\title{
A Vortical Hot Tower Route to Tropical Cyclogenesis
}

\author{
M. T. Montgomery, M. E. Nicholls, T. A. Cram, and A. B. Saunders \\ Department of Atmospheric Science, Colorado State University, Fort Collins, Colorado
}

(Manuscript received 5 December 2003, in final form 22 February 2005)

\begin{abstract}
A nonhydrostatic cloud model is used to examine the thermomechanics of tropical cyclogenesis under realistic meteorological conditions. Observations motivate the focus on the problem of how a midtropospheric cyclonic vortex, a frequent by-product of mesoscale convective systems during summertime conditions over tropical oceans, may be transformed into a surface-concentrated (warm core) tropical depression. As a first step, the vortex transformation is studied in the absence of vertical wind shear or zonal flow.

Within the cyclonic vorticity-rich environment of the mesoscale convective vortex (MCV) embryo, the simulations demonstrate that small-scale cumulonimbus towers possessing intense cyclonic vorticity in their cores [vortical hot towers (VHTs)] emerge as the preferred coherent structures. The VHTs acquire their vertical vorticity through a combination of tilting of MCV horizontal vorticity and stretching of MCV and VHT-generated vertical vorticity. Horizontally localized and exhibiting convective lifetimes on the order of $1 \mathrm{~h}$, VHTs overcome the generally adverse effects of downdrafts by consuming convective available potential energy in their local environment, humidifying the middle and upper troposphere, and undergoing diabatic vortex merger with neighboring towers.

During metamorphosis, the VHTs vortically prime the mesoscale environment and collectively mimic a quasi-steady diabatic heating rate within the MCV embryo. A quasi-balanced toroidal (transverse) circulation develops on the system scale that converges cyclonic vorticity of the initial MCV and small-scale vorticity anomalies generated by subsequent tower activity. The VHTs are found to accelerate the spinup of near-surface mean tangential winds relative to an approximate axisymmetric model that excises the VHTs. This upscale growth mechanism appears capable of generating a tropical depression vortex on time scales on the order of 1-2 days, for reasonable parameter choices.

Further tests of the VHT paradigm are advocated through diagnoses of operational weather prediction models, higher resolution simulations of the current configuration, examination of disruption scenarios for incipient vortices, and a meteorological field experiment.
\end{abstract}

\section{Introduction}

\section{a. Motivation}

Tropical cyclones (TCs) form typically in association with mesoscale convective systems near the trough (low pressure) region of synoptic-scale tropical weather disturbances, such as easterly waves (Gray 1968, 1998; Cotton and Anthes 1989; Raymond et al. 1998, and references included within). Early work hypothesized that cumulonimbus convection cooperated with the larger scales in a (axisymmetric) positive feedback process (Ooyama 1964; Charney and Eliassen 1964). While

Corresponding author address: Dr. Michael T. Montgomery, Dept. of Atmospheric Science, Colorado State University, Fort Collins, CO 80523.

E-mail: mtm@atmos.colostate.edu purporting to predict the growth rate of smallamplitude mesoscale vortices, these approaches tended to be disguised descriptions of cumulus convection, with the largest growth rates occurring at the smallest horizontal scales (Ooyama 1982, section 5, p. 378; cf. Lilly 1960). This "ultraviolet catastrophe" was partially side stepped when a (axisymmetric) wind-induced surface heat exchange (WISHE) process was demonstrated to be the primary mechanism for amplification of a finite amplitude surface-concentrated (tropical depression) vortex (Ooyama 1969; Rotunno and Emanuel 1987), with cumulus convection transporting the heat extracted at the ocean surface to the upper troposphere. ${ }^{1}$ The problem of how a synoptic-scale tropical disturbance may be transformed into a surface-concen-

\footnotetext{
${ }^{1}$ See Smith (1997) for a contemporary review.
} 
trated (warm core) mesoscale tropical depression vortex has been an enigma of tropical and dynamical meteorology. This transformation will be hereafter referred to as tropical cyclogenesis.

Observations suggest that cyclonic mesoscale convective vortices (MCVs) forming in the stratiform precipitation region near midlevels of the troposphere in the trough region of tropical disturbances, are generic precursors to TC genesis in the deep Tropics (e.g., Zehr 1992; Mapes and Houze 1995; Harr and Elsberry 1996; Harr et al. 1996; Bister and Emanuel 1997, hereafter BE97; Ritchie and Holland 1997, hereafter RH97; Raymond et al. 1998; Reasor et al. 2005). Interpretations differ, however, as to how MCVs contribute to the formation of a surface-concentrated tropical depression vortex (BE97; Montgomery and Enagonio 1998, hereafter ME98; Raymond et al. 1998; Ritchie 2003; Molinari et al. 2004; Reasor et al. 2005).

\section{b. Hot towers revisited}

In their pioneering study, Riehl and Malkus (1958) implicated the role of hot towers (horizontally small but intense cumulonimbus convection cores that reach the tropopause via nearly undilute ascent) in the vertical heat transport and mass flux of the tropical overturning circulation (Hadley cell). There is now little doubt that deep cumulonimbus convection is the dominant mode of vertical mass and energy transport in the tropical troposphere, but comparatively little quantitative data are available for the hot tower-like structures that are observed to exist in pre-TC environments.

Simpson et al. (1998) and Ritchie et al. (2002) suggested that hot towers contribute to TC genesis and intensification via subsidence warming around them and the attending hydrostatic surface pressure fall. A different hypothesis was proposed by ME98, Möller and Montgomery (2000, hereafter MM00), and Enagonio and Montgomery (2001, hereafter EM01). Using idealized quasigeostrophic, asymmetric balance, and shallow water primitive equation models, these studies suggested that vortex merger and vortex axisymmetrization processes accompanying the diabatic generation of near-surface $(0<z<2 \mathrm{~km})$ cyclonic potential vorticity (PV) anomalies could intensify a seedling vortex to tropical storm-strength vortex on realistic time scales.

\section{c. Cloud-resolving simulations furnish new insight}

To understand further the vorticity dynamics of the incipient organization phase Hendricks et al. (2004, hereafter H04) extended the genesis study of Davis and
Bosart (2001) by analyzing a high-resolution nearcloud-resolving numerical simulation of Hurricane Diana (1984) using a 3-km horizontal grid spacing. The H04 study suggested that convective plumes with intense vertical vorticity in their cores are the preferred convective structures. The simulated plumes forming in a cyclonic vorticity rich environment acted to generate multiple small-scale $[O(10-30 \mathrm{~km})]$ intense cyclonic vortex tubes. These intense cyclonic vorticity cores possess an intrinsic centrifugal and vortex-Rossby elasticity that tends to protect them from detrimental effects of lateral entrainment (McWilliams 1984; McIntyre 1993; Montgomery and Kallenbach 1997; Julien et al. 1999; Reasor et al. 2004). H04 coined the term vortical hot towers (VHTs) to describe these coherent structures (cf. Anthes 2003). In the favorable pre-Diana environment, the generation of VHTs led to multiple mergers/ axisymmetrization of the VHTs and a corresponding upscale vorticity cascade to the tropical storm scale. While broadly verifying the thought experiments of ME98, MM00, and EM01, the H04 analysis provided new insight into the convective/vorticity dynamics that were not previously anticipated.

The H04 study suggested a two-stage process for creating the near-surface tropical storm vortex that eventually became Hurricane Diana. The first phase was a vortical preconditioning of the lower troposphere by the generation of multiple VHTs and their competition with each other for ambient convective available potential energy (CAPE) and angular momentum (cf. Ooyama 1982). The VHTs were found to have relatively short convective lifetimes (order $1 \mathrm{~h}$ ) and to possess maximum tangential velocities near the ocean surface at the time of their maximum intensity. The second phase consisted of multiple vortex mergers and axisymmetrization of the VHTs, broadly resembling quasitwo-dimensional turbulence and vortex dynamics phenomenology (e.g., McWilliams 1984; Melander et al. 1988; Guinn and Schubert 1993; ME98; MM00; EM01). Unlike inviscid and adiabatic vortex merger dynamics, however, the diabatically and frictionally forced toroidal (secondary) circulation operating in the vicinity of the VHTs enhances the near-surface $(0<z<2 \mathrm{~km})$ inflow, and the VHTs typically merge near the surface first. $^{2}$ The diabatic concentration of PV substance (Haynes and McIntyre 1987) at these levels produces an intensification of near-surface PV. H04 coined this

\footnotetext{
${ }^{2}$ The surface bias in the merger of VHTs is similar to numerical experiments by Wang and Holland (1995) examining the merger of full-fledged hurricanes.
} 
diabatically modified merger process as a diabatic vortex merger.

\section{d. Working hypotheses}

The H04 study suggests a new paradigm of TC genesis within a vorticity-rich environment via VHTs and their aggregate effects. Underlying the paradigm are two main hypotheses:

(a) There exists sufficient CAPE and low-level cyclonic relative vorticity in pre-TC environments to initiate the formation of the VHTs by deep convective processes.

(b) VHTs are the preferred convective structures during the incipient formation phase and serve as the primary building blocks of the mesoscale cyclonic vortex.

\section{e. Current approach}

Motivated by the observations cited above, we examine here the role of VHTs in the metamorphosis from a midlevel cyclonic MCV to a tropical depression. For simplicity, the initial MCVs used are axisymmetric; they possess their maximum vertical vorticity near the $z=$ 4-5-km level; and they have a relative surface vorticity of $O\left(1-2 \times 10^{-4} \mathrm{~s}^{-1}\right)$. The numerical simulations commence with a favorable kinematic environment (i.e., zero ambient mean flow) and a favorable thermodynamic environment [i.e., a conditionally unstable troposphere overlying a warm ocean with sea surface temperature $(\mathrm{SST}) \geq 26^{\circ} \mathrm{C}$.

Using a 2- or 3-km horizontal grid spacing on the fine mesh of the model, it will be shown that in a conditionally unstable environment, a single midlevel cyclonic MCV can undergo a metamorphosis to a tropical depression vortex on meteorologically realistic (order 1-2 days) time scales. The metamorphosis is demonstrated to occur here in association with the generation of intense VHTs within the cyclonic vorticity-rich environment of the initial MCV, mid-to-upper-level moistening by the VHTs, diabatic mergers between VHTs, and the near-surface and mid- to upper-level inflow on the system scale that is induced by the ensemble of VHTs.

The paper is organized as follows: section 2 summarizes the nonhydrostatic cloud model setup and defines the initial MCV, temperature, and moisture profiles used for the array of idealized numerical experiments presented. Section 3 highlights the control and sensitivity experiments. Section 4 examines some thermodynamical aspects of the metamorphosis process. The early-time dynamics of VHTs is examined in section 5. Diabatic vortex merger phenomenology associated with the VHTs is summarized in section 6 . The influence of the VHTs on the system-scale flow is considered in section 7. Section 8 summarizes the main findings.

\section{Model and initial conditions}

\section{a. Cloud model summary}

For the numerical simulations we employ the Regional Atmospheric Modeling System (RAMS), developed at Colorado State University (Pielke et al. 1992; Cotton et al. 2003). The RAMS is a three-dimensional nonhydrostatic numerical modeling system comprising time-dependent equations for velocity, nondimensional pressure perturbation, ice-liquid water potential temperature (Tripoli and Cotton 1981), total water mixing ratio, and cloud microphysics. Vapor mixing ratio and potential temperature are diagnostic [see Tripoli and Cotton (1982) for details]. The microphysics scheme developed by Walko et al. (1995) has categories for cloud droplets, rain, pristine ice, snow, aggregates, graupel, and hail. The surface parameterization of heat, vapor, and momentum fluxes is based on the Louis (1979) scheme. The roughness length over water is dependent on the surface wind speed according to the relation derived by Charnock (1955). The longwave and shortwave radiation scheme developed by Harrington (1997) is employed, which includes interactions with cloud hydrometeors. A standard first-order subgrid-scale turbulence scheme developed by Smagorinsky (1963) is used, with modifications by Lilly (1962) and Hill (1974). These modifications enhance diffusion in unstable conditions and reduce diffusion in stable conditions.

RAMS utilizes the two-way interactive multiple nested grid scheme developed by Clark and Farley (1984). This allows explicit representation of cloudscale features within the finest grid while enabling a large domain size to be used, thereby minimizing the impact of lateral boundary conditions. A standard radiation boundary condition discussed by Klemp and Wilhelmson (1978) is used at the lateral boundaries, which assumes that disturbances reaching the boundaries move as linearly propagating gravity waves. A standard Rayleigh friction layer is included at upper levels in order to minimize reflection of gravity waves from the top of the model. Unless otherwise stated, all microphysical, radiative, and diffusive parameters employed in our RAMS simulations are the standard ones employed in version 4.29 for tropical summer conditions. To facilitate reproducibility and/or side-by-side comparisons with other cloud modeling systems, appendix A provides further details of the model configuration and numerical parameters.

For all numerical experiments three grids are used. 
TABLE 1. RAMS genesis experiments-initial conditions.

\begin{tabular}{|c|c|c|c|c|}
\hline No. & Name & $\begin{array}{c}\mathrm{RMW} \\
(\mathrm{km})\end{array}$ & $\begin{array}{l}\operatorname{Max} v \\
\left(\mathrm{~m} \mathrm{~s}^{-1}\right)\end{array}$ & Notes \\
\hline A1 & Control & 75 & $6.6 @ 4$ km & $\begin{array}{l}\Delta x=\Delta y=2 \mathrm{~km}, \mathrm{SST}=29^{\circ} \mathrm{C} . \text { Metamorphosis to surface vortex successful. Becomes } \\
\text { miniature tropical cyclone by approximately } 60 \mathrm{~h} \text {. Mean near-surface tangential wind } \\
\quad \approx 12 \mathrm{~m} \mathrm{~s}^{-1} \text { at } 24 \mathrm{~h} \text {, and } 46 \mathrm{~m} \mathrm{~s}^{-1} \text { at } 72 \mathrm{~h} .\end{array}$ \\
\hline $\mathrm{A} 2$ & $3 \mathrm{~km}$ & 75 & $6.6 @ 4$ km & $\begin{array}{l}\text { Same as control, except } \Delta x=\Delta y=3 \mathrm{~km} \text {. Metamorphosis to surface vortex successful. } \\
\text { Mean near-surface tangential wind } \approx 13 \mathrm{~m} \mathrm{~s}^{-1} \text { at } 24 \mathrm{~h} \text {, and } 46 \mathrm{~m} \mathrm{~s}^{-1} \text { at } 72 \mathrm{~h} \text {. }\end{array}$ \\
\hline B1 & No bubble & 75 & $6.6 @ 4$ km & $\begin{array}{l}\text { Same as control, except } \Delta x=\Delta y=3 \mathrm{~km} \text { and no initial convective anomaly used. } \\
\text { Metamorphosis as in expt A1 once deep convection ensues at } t \approx 14 \mathrm{~h} .\end{array}$ \\
\hline B2 & $\begin{array}{l}\text { cape-less } \\
(2 \mathrm{~km})\end{array}$ & 75 & $6.6 @ 4$ km & $\begin{array}{l}\text { Same as control, except } \Delta x=\Delta y=2 \mathrm{~km} \text {. Low-level moisture decreased by } 2 \mathrm{~g} \mathrm{~kg}^{-1} \text {. } \\
\text { Metamorphosis successful, but slower rate of development. Mean near-surface } \\
\text { tangential wind } \approx 10 \mathrm{~m} \mathrm{~s}^{-1} \text { at } 48 \mathrm{~h} \text {. }\end{array}$ \\
\hline B3 & $\begin{array}{l}\text { cape- less } \\
(3 \mathrm{~km})\end{array}$ & 75 & $6.6 @ 4$ km & $\begin{array}{l}\text { Same as control, except } \Delta x=\Delta y=3 \mathrm{~km} \text {. Low-level moisture decreased by } 2 \mathrm{~g} \mathrm{~kg}^{-1} \text {. } \\
\text { Metamorphosis successful, but slower rate of development. Mean near-surface } \\
\text { tangential wind } \approx 9 \mathrm{~m} \mathrm{~s}^{-1} \text { at } 48 \mathrm{~h} \text {. }\end{array}$ \\
\hline B4 & $\begin{array}{l}\text { cape more } \\
\text { stable }\end{array}$ & 75 & $6.6 @ 4$ km & $\begin{array}{l}\text { Same as control, except } \Delta x=\Delta y=3 \mathrm{~km} \text {, and surface-based CAPE reduced by } \\
\text { increasing temperature aloft by approx } 2 \mathrm{~K} \text {. Metamorphosis successful, but slower } \\
\text { development. Mean near-surface tangential wind } \approx 15 \mathrm{~m} \mathrm{~s}^{-1} \text { at } 48 \mathrm{~h} \text {. }\end{array}$ \\
\hline B5 & sst 26 & 75 & $6.6 @ 4$ km & $\begin{array}{l}\text { Same as control, except } \Delta x=\Delta y=3 \mathrm{~km} \text {, and SST }=26^{\circ} \mathrm{C} \text {. Metamorphosis successful. } \\
\text { Mean near-surface tangential wind } \approx 10 \mathrm{~m} \mathrm{~s}^{-1} \text { at } 48 \mathrm{~h} \text {, tropical storm strength at } \\
72 \mathrm{~h} \text {. }\end{array}$ \\
\hline B6 & sst 28 & 75 & $6.6 @ 4$ km & $\begin{array}{l}\text { Same as control, except } \Delta x=\Delta y=3 \mathrm{~km} \text {, and SST }=28^{\circ} \mathrm{C} \text {. Metamorphosis successful. } \\
\text { Formation of miniature TC with mean near-surface tangential winds } \approx 40 \mathrm{~m} \mathrm{~s}^{-1} \text { at } \\
72 \mathrm{~h} \text {. }\end{array}$ \\
\hline B7 & $\begin{array}{l}\text { center } \\
\text { bubble }\end{array}$ & 75 & $6.6 @ 4$ km & $\begin{array}{l}\text { Same as control, except } \Delta x=\Delta y=3 \mathrm{~km} \text {, and initial warm convective anomaly located } \\
\text { under center of midlevel MCV. Metamorphosis successful. }\end{array}$ \\
\hline B8 & $\begin{array}{l}\text { broad } \\
\text { moist }\end{array}$ & 75 & $6.6 @ 4$ km & $\begin{array}{l}\text { Same as control, except } \Delta x=\Delta y=3 \mathrm{~km} \text {, and low-level moisture anomaly increased } \\
\text { from } 100 \text { - to } 200-\mathrm{km} \text { radius. Metamorphosis successful. Mean near-surface tangential } \\
\text { wind } \approx 23 \mathrm{~m} \mathrm{~s}^{-1} \text { at } 48 \mathrm{~h} \text {, hurricane at } 72 \mathrm{~h} \text {. }\end{array}$ \\
\hline B9 & radiation & 75 & $6.6 @ 4$ km & $\begin{array}{l}\text { Same as control, except } \Delta x=\Delta y=3 \mathrm{~km} \text {, and model initialized } 12 \mathrm{~h} \text { later to investigate } \\
\text { diurnal effect of radiation. Metamorphosis successful, as in expt A1. Intensification to } \\
\text { hurricane strength vortex by } 72 \mathrm{~h} \text {. }\end{array}$ \\
\hline $\mathrm{C} 1$ & no vortex & - & - & No initial vortex. No surface development whatsoever. \\
\hline $\mathrm{C} 2$ & big rmw & 100 & $6.6 @ 4$ km & $\begin{array}{l}\text { Same as control, except } \Delta x=\Delta y=3 \mathrm{~km} \text {, and radius of initial vortex increased to } \\
100 \mathrm{~km} \text {. Metamorphosis successful. Mean near-surface tangential wind } \approx 22 \mathrm{~m} \mathrm{~s}^{-1} \text { at } \\
48 \mathrm{~h} . \mathrm{RMW}=20 \mathrm{~km} \text { at } 48 \mathrm{~h} \text {. }\end{array}$ \\
\hline $\mathrm{C} 3$ & weak vortex & 75 & $5.0 @ 4$ km & $\begin{array}{l}\text { Same as control, except } \Delta x=\Delta y=3 \mathrm{~km} \text {, and maximum tangential velocity of initial } \\
\text { vortex decreased to } 5.0 \mathrm{~m} \mathrm{~s}^{-1} \text {. Metamorphosis successful, but slower rate of } \\
\text { development. Mean near-surface tangential wind } \approx 9 \mathrm{~m} \mathrm{~s}^{-1} \text { at } 72 \mathrm{~h} \text {. Circulation very } \\
\text { asymmetric even at } 72 \mathrm{~h} \text {. }\end{array}$ \\
\hline $\mathrm{C} 4$ & $\begin{array}{l}\text { weak small } \\
\text { vortex }\end{array}$ & 50 & $5.0 @ 4$ km & $\begin{array}{l}\text { Same as experiment } \mathrm{C} 3 \text { except RMW }=50 \mathrm{~km} \text {. Metamorphosis successful, but as in } \\
\text { expt C3 slow rate of development. Mean near-surface tangential wind } \approx 8 \mathrm{~m} \mathrm{~s}^{-1} \text { at } \\
48 \mathrm{~h} \text {. Very asymmetric circulation at } 72 \mathrm{~h} \text {. }\end{array}$ \\
\hline $\mathrm{C} 5$ & raised vortex & 75 & $6.6 @ 5$ km & $\begin{array}{l}\Delta x=\Delta y=2 \mathrm{~km} \text {. Metamorphosis successful, but slow rate of development. Mean } \\
\text { near-surface tangential wind } \approx 12 \mathrm{~m} \mathrm{~s}^{-1} \text { at } 48 \mathrm{~h} \text {. Asymmetric circulation at } 72 \mathrm{~h} \text {. }\end{array}$ \\
\hline D1 & no tfz qfz & 75 & $6.6 @ 4$ km & $\begin{array}{l}\text { Same as control, except } \Delta x=\Delta y=3 \mathrm{~km} \text {, and surface sensible and latent heat fluxes } \\
\text { turned off. Metamorphosis successful. Mean near-surface tangential wind } \approx 12 \mathrm{~m} \mathrm{~s}^{-1} \\
\text { at } 24 \mathrm{~h} \text {. No subsequent intensification. }\end{array}$ \\
\hline D2 & no mtm flux & 75 & $6.6 @ 4$ km & $\begin{array}{l}\text { Same as control, except } \Delta x=\Delta y=3 \mathrm{~km} \text {, and surface momentum fluxes turned off. } \\
\text { Metamorphosis successful, but surface development slower than expt A1. Continued } \\
\text { intensification to minimal tropical storm strength vortex at } 72 \mathrm{~h} \text { with an RMW = } \\
70 \mathrm{~km} \text {. }\end{array}$ \\
\hline E1 & zero Coriolis & 75 & $6.6 @ 4$ km & $\begin{array}{l}\text { Same as control, except } \Delta x=\Delta y=3 \mathrm{~km} \text {, and Coriolis parameter set to zero }(f=0) \text {. } \\
\text { Metamorphosis successful. Develop surface-concentrated vortex as in control, but no } \\
\text { subsequent intensification observed through } 72 \mathrm{~h} \text {. }\end{array}$ \\
\hline
\end{tabular}

For the control experiment (expt A1, see Table 1) the horizontal grid increments are 24,6 , and $2 \mathrm{~km}$, with $(x$, $y, z$ ) dimensions of $64 \times 64 \times 26,90 \times 90 \times 26$, and 137 $\times 137 \times 26$, respectively. Each nested grid is centered within the next coarsest grid. The vertical grid increment is $400 \mathrm{~m}$ at the surface and gradually stretched with height to the top of the domain at $22 \mathrm{~km}$. The depth of the Rayleigh friction layer is $5 \mathrm{~km}$. 

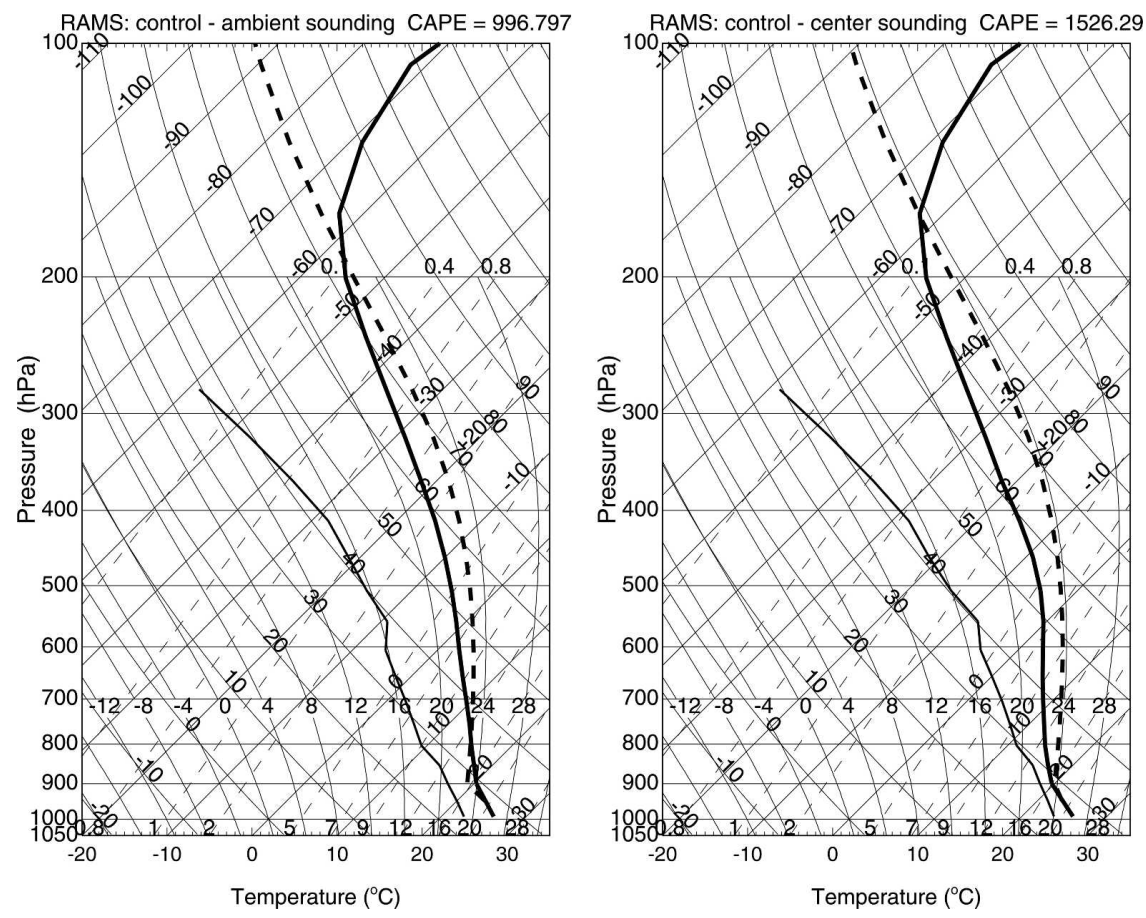

FIG. 1. Skew $T$ - $\log p$ atmospheric sounding plots at the domain center and ambient environment for the control experiment (expt A1). The temperature sounding is the thick solid line, dewpoint temperature is the thin solid line, and lifted parcel ascent path is the dashed line. Mixed-layer CAPE values for the lowest $1 \mathrm{~km}$ are indicated.

\section{b. Initial temperature and moisture profiles}

The initial temperature structure is the mean Atlantic hurricane season sounding (Jordan 1958). This sounding is representative of non-Saharan-air-layer air (Dunion and Velden 2004). With an SST of $29^{\circ} \mathrm{C}$ (expt A1: control experiment) the sounding contains approximately $997 \mathrm{~J} \mathrm{~kg}^{-1}$ of 1-km mixed-layer CAPE based on pseudoadiabatic ascent (Fig. 1: RAMS control-ambient sounding). Sensitivity experiments using lower SSTs (e.g., $26^{\circ} \mathrm{C}$, expt B5; $28^{\circ} \mathrm{C}$, expt B6) furnish lower values of mixed-layer CAPE, yet exhibit similar qualitative behavior to the control (Table 1).

For the control experiment, and the majority of the sensitivity experiments (Table 1 ), the low- to midtroposphere $(z<6 \mathrm{~km})$ is moistened near the center of the initial MCV. The moisture enhancement is assumed to be the result of enhanced sea-to-air moisture fluxes in association with the weak MCV surface circulation. The moisture anomaly has a mixing ratio $q_{v}$ surplus of approximately $1.3 \mathrm{~g} \mathrm{~kg}^{-1}$ on $z=0$ near the $\mathrm{MCV}$ center (see Fig. 2d). For the control experiment, the low-level moisture enhancement increases the 1-km mixed-layer CAPE to $1526 \mathrm{~J} \mathrm{~kg}^{-1}$ at the domain center (Fig. 1, RAMS control-center sounding).

\section{c. Why initialize with a single MCV?}

The present approach is considered complementary to prior work studying the transformation of an easterly wave into a TC using a hydrostatic primitive equation model with cumulus parameterization (Kurihara and Tuleya 1981). A typical easterly wave in the African jet has a horizontal wavelength of approximately $2500 \mathrm{~km}$ (e.g., Reed et al. 1977) and spawns mesoscale convective systems (MCSs) near the trough region of the wave. Tropical cyclone formation is generally believed to occur in association with one or more MCVs that are a by-product of the MCSs (e.g., BE97; Raymond et al. 1998; Gray 1998). Rather than simulate this complex multiscale environment, for simplicity, the experiments here commence with a single MCV that is initially in hydrostatic and gradient wind balance. This shortcut assumes that an MCS has already created an MCV and enables one to investigate in detail the convective/ vorticity dynamics that ensue within this local environment using a near cloud-resolving model.

\section{d. The initial $M C V$}

The initialization of pressure, temperature, and winds follows the procedure presented by Nicholls et al. 

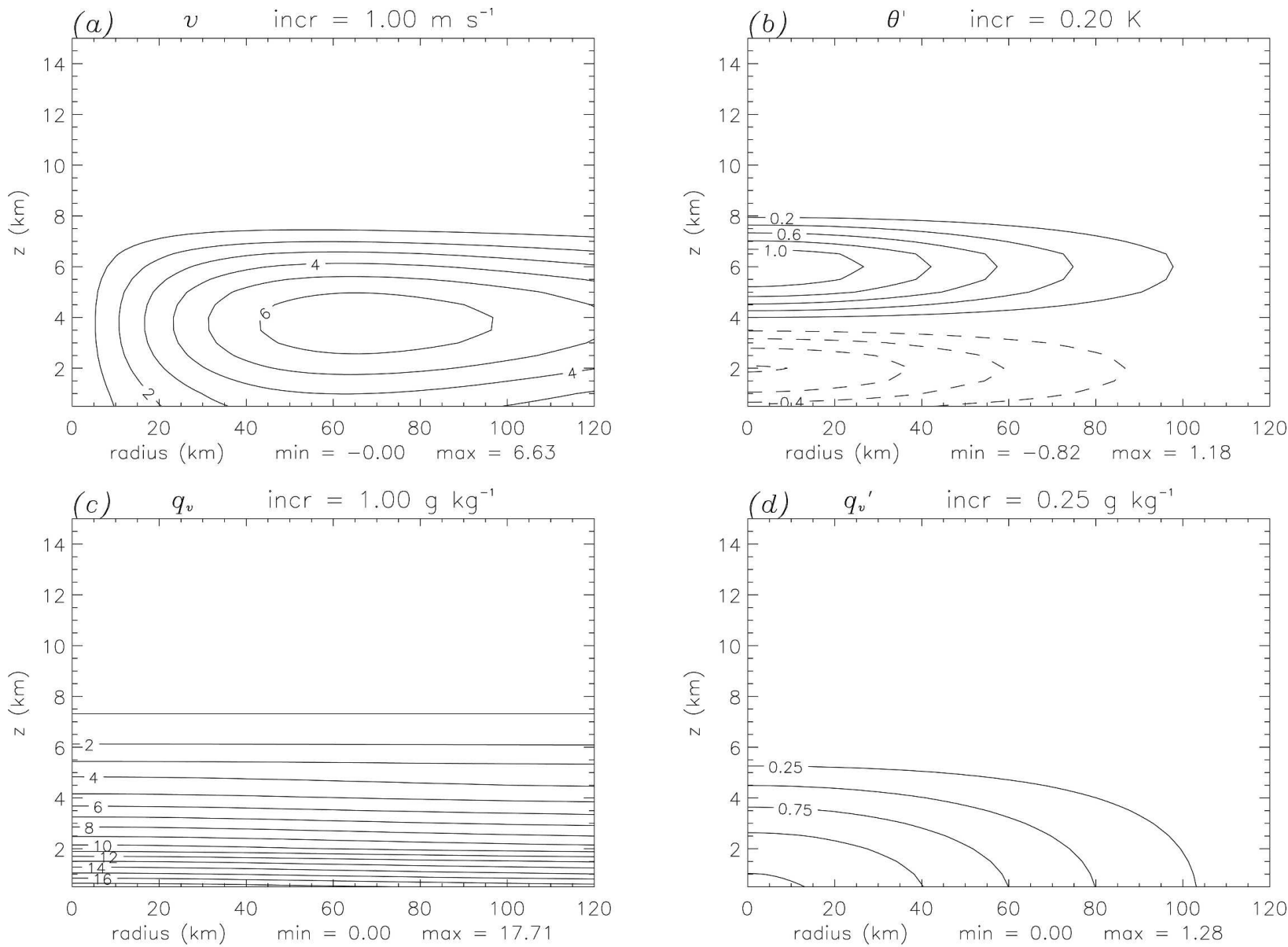

(e) $\quad \eta \quad$ incr $=5.00 E-05 \mathrm{~s}^{-1}$
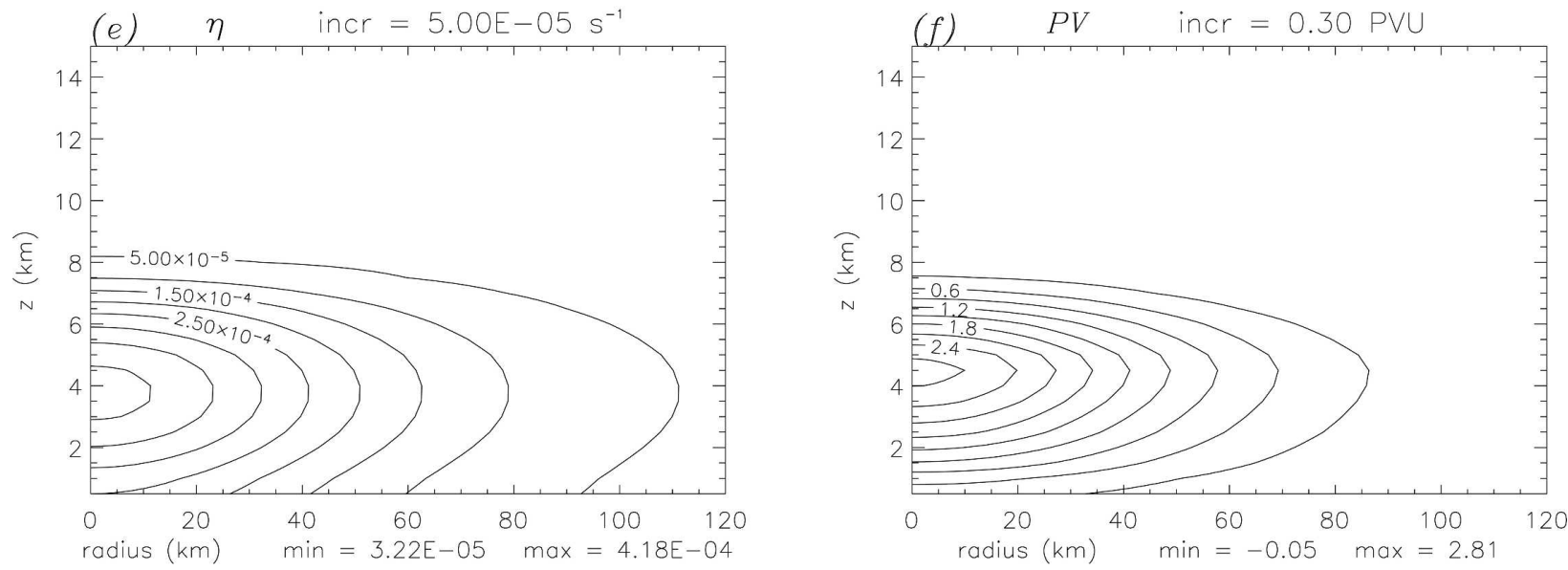

FIG. 2. Initial conditions used for the control experiment, expt A1. Shown are azimuthal averages of (a) tangential wind, (b) potential temperature anomaly from ambient, (c) total water vapor mixing ratio, (d) water vapor mixing ratio anomaly from ambient, (e) absolute vertical vorticity, and (d) Rossby-Ertel PV in PVU (1 PVU $\left.=1.0 \times 10^{-6} \mathrm{~m}^{2} \mathrm{~s}^{-1} \mathrm{~K} \mathrm{~kg}^{-1}\right)$ of Hoskins et al. (1985).

(2000). The perturbation form of the hydrostatic equation can be written as follows:

$$
\frac{\partial \pi^{\prime}}{\partial z}=B
$$

where $\pi^{\prime}$ is a perturbation of the Exner function defined by $c_{p}\left(p / p_{r}\right)^{\kappa}, c_{p}$ is the specific heat of dry air at constant pressure, $p$ is total pressure, $p_{r}$ is a reference pressure, and $\kappa=R / c_{p}$ with $R$ the dry air gas constant per unit mass; $B=g \theta_{v}^{\prime} / \theta_{v_{o}}^{2}$, with $\theta_{v_{o}}$ the initial unper- 
turbed horizontally homogeneous virtual potential temperature, $\theta_{v}^{\prime}$ the perturbed virtual potential temperature, and $g$ the acceleration because of gravity. The function $B$ is specified by

$$
B=\frac{B_{0} a^{2}}{\left(a^{2}+r^{2}\right)}\left[\alpha \sin \left(\frac{\pi z}{H}\right)-\beta \sin \left(\frac{2 \pi z}{H}\right)\right]
$$

where $r$ is the radius, $a$ is a constant that is approximately equal to the radius of maximum wind, and $H$ is the height of the vortex. Specifying $B$ rather than the buoyancy $B \theta_{v_{o}}$ allows Eq. (1) to be integrated exactly with respect to $z$ to obtain an analytical expression for $\pi^{\prime}$, for arbitrary $\theta_{v_{o}}(z)$. Using the condition that $\pi^{\prime}=0$ at $z=H$ gives

$$
\begin{aligned}
\pi^{\prime}= & \frac{B_{0} a^{2} H}{\left(a^{2}+r^{2}\right) \pi}\left\{-\alpha\left[\cos \left(\frac{\pi z}{H}\right)+1\right]\right. \\
& \left.+\frac{\beta}{2}\left[\cos \left(\frac{2 \pi z}{H}\right)-1\right]\right\} .
\end{aligned}
$$

The tangential velocity $v$ is assumed to be in gradient wind balance, giving

$$
v=-\frac{r f}{2}+\sqrt{\left(\frac{r f}{2}\right)^{2}-\frac{2 r^{2} \theta_{v_{o}} \pi^{\prime}}{\left(r^{2}+a^{2}\right)}},
$$

where $f$ is the constant Coriolis parameter. For the control simulation $H=8 \mathrm{~km}, a=75 \mathrm{~km}, \alpha=0.2, \beta=1$. These values of $H, \alpha$, and $\beta$ furnish a vortex with maximum tangential winds at a height of $4 \mathrm{~km}$ above sea level. The value of $B_{0}$ was chosen to give a maximum tangential velocity of $6.6 \mathrm{~m} \mathrm{~s}^{-1}$ (see Fig. 2a). The latitude at which the Coriolis parameter was calculated was taken to be $15^{\circ} \mathrm{N}$. The mean vortex Rossby number $R=v / f r$ associated with the initial MCV is $\sim 2$ at the radius of maximum tangential wind (RMW). The initial vortex shown in Fig. 2a is broadly consistent with airborne Doppler radar observations of the pre-Dolly MCV (1996; Reasor et al. 2005) and the pre-TC MCVs observed during the Tropical Experiment in Mexico (TEXMEX; Raymond et al. 1998, their Figs. 10b, 11b, $14 b$, and 15b).

The radius-height distribution of absolute vertical vorticity and Rossby-Ertel PV (Hoskins et al. 1985) for this MCV is shown in Figs. 2e,f. Since the product of twice the absolute (vertical) rotation rate $(f+2 v / r)$ and Rossby-Ertel PV is everywhere positive below $z=8$ $\mathrm{km}$, the initial vortex is symmetrically stable to dry displacements (e.g., Shapiro and Montgomery 1993). All simulations using the initial MCV moreover exhibit no evidence of baroclinc instability. ${ }^{3}$

\section{Experimental results}

\section{a. Results for control experiment}

The principal features of the control simulation (expt A1, Table 1) are summarized here. For this experiment, a local heating was applied for $300 \mathrm{~s}$ in order to create a warm bubble (temperature surplus $2 \mathrm{~K}$ ) at low levels $(z=2 \mathrm{~km}), 50 \mathrm{~km}$ to the east of the MCV center. The bubble method serves to stimulate cumulus convection in the local environment of the MCV. ${ }^{4}$

Snapshots of the absolute vertical vorticity $f+\zeta$ and diabatic heating rate $\dot{\theta}$ are shown in Fig. 3 at $t=7 \mathrm{~h}$, early into the vortex metamorphosis process. Horizontal surfaces correspond to $z=1,4$, and $7 \mathrm{~km}$. Color bars indicate the magnitude of $f+\zeta$ and $\dot{\theta}$, respectively. Light green and dark blue regions correspond to the cyclonic absolute vorticity from the initial MCV; light green corresponds to absolute vorticity between $2.5 \times$ $10^{-4} \mathrm{~s}^{-1}$ and $5.0 \times 10^{-4} \mathrm{~s}^{-1}$; dark blue corresponds to absolute vorticity between zero and $2.5 \times 10^{-4} \mathrm{~s}^{-1}$. The VHTs (orange and red regions) are located within the light green regions. The VHTs are consuming ambient CAPE at this time and exhibit an approximate collocation of regions exhibiting intense absolute cyclonic vorticity $\left(\eta>3 \times 10^{-3} \mathrm{~s}^{-1}\right)$ and diabatic heating rates $(\dot{\theta}>$ $\left.160 \mathrm{~K} \mathrm{~h}^{-1}\right)$. Although the two VHTs illustrated here merge soon after the time shown (Figs. 12-15 and section 6 provides further details of this merger event), the

\footnotetext{
${ }^{3}$ This is unlike the companion problem of rotating oceanic convection arising within preconditioned environments associated with strong (baroclinically unstable) cold core eddies in the Labrador or Greenland seas during winter (e.g., Legg et al. 1998; Molemaker and Dykstra 2000). In the Labrador/Greenland sea problems, the baroclinic instability growth rates are significant and the ratio of horizontal eddy scale to the ambient Rossby deformation radius is of order unity, much larger than the corresponding ratio in the TC genesis problem. The coupling between baroclinic instabilities and convective plumes is thus important in the cold core ocean eddy problem, but negligible in the TC genesis problem. Tropical cyclone genesis has complexities without ocean analog, associated with strong phase transitions (latent heat of condensation and evaporative cooling), intense (large Rossby number) VHTs, convective downdrafts, spreading cold pools, etc.

${ }^{4}$ In real situations, an elevated MCV is usually subject to some low- to midlevel vertical wind shear. If the shear is not strong enough to tear the MCV apart (e.g., Reasor et al. 2004), the shear will stimulate convection near the radius of maximum tangential velocity in the downshear direction (Ritchie and Holland 1997, p. 1380; Trier et al. 2000). The warm bubble method may thus be regarded as a crude convective response for an elevated $\mathrm{MCV}$ subject to a brief episode of weak vertical shear.
} 

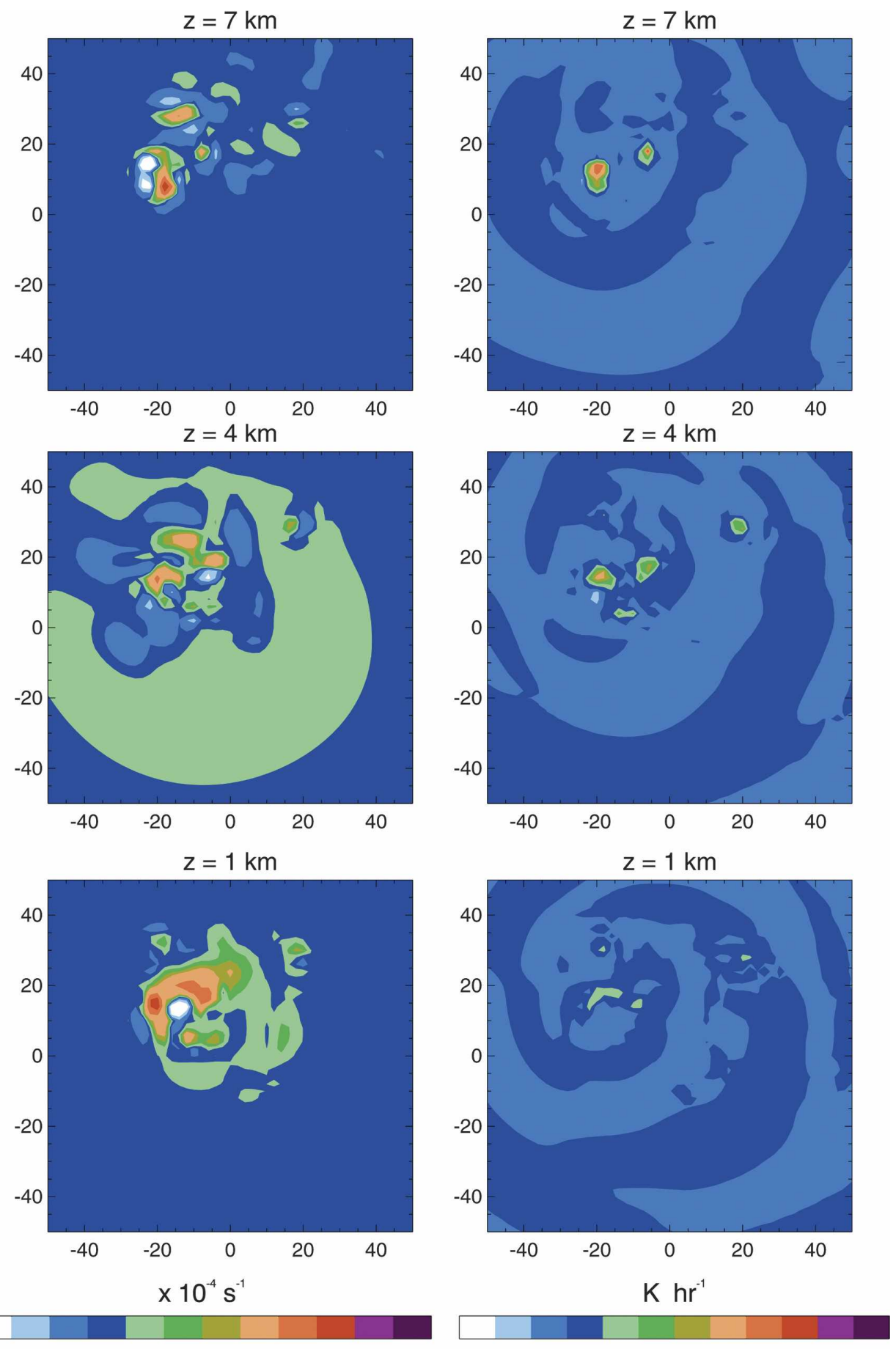

$\begin{array}{lllllllllll}-10 & -5 & 0 & 2.5 & 5 & 7.5 & 10 & 20 & 30 & 40 & 50\end{array}$

$-80-40 \quad 0 \quad 40 \quad 80120160200240280320$

FIG. 3. (left) Absolute vorticity $f+\zeta$ in units of $10^{-4} \mathrm{~s}^{-1}$ and (right) diabatic heating rate $\dot{\theta}$ in units of $\mathrm{K} \mathrm{h}^{-1}$ on horizontal surfaces $z=1,4$, and $7 \mathrm{~km}$ at $t=7 \mathrm{~h}$ into the control simulation, expt A1. Axes are in $\mathrm{km}$.

resulting VHT complex eventually dies, leaving behind a heightened low-level cyclonic vertical vorticity anomaly. After several such convective cycles, the nearsurface $(0<z<2 \mathrm{~km})$ mesoscale circulation intensifies to a point where an explosive amplification of the lowlevel tangential winds is observed $(t \approx 60 \mathrm{~h})$, a rapid intensification phase consistent with WISHE amplification (Rotunno and Emanuel 1987). 
Figure 4 depicts the azimuthal mean radius-height structure of the tangential velocity field and potential temperature deviation from the environment over the first $24 \mathrm{~h}$. By $12 \mathrm{~h}$, a weak tropical depression vortex has formed with an attending warm core structure, as evidenced by a low-level vortex with maximum azimuthal mean tangential velocity at $z=1 \mathrm{~km}$ of approximately $7 \mathrm{~m} \mathrm{~s}^{-1}$ and a positive potential temperature anomaly in the upper troposphere $(6<z<10 \mathrm{~km}$; Figs. $4 \mathrm{c}$,d). By $24 \mathrm{~h}$, the maximum surface winds have intensified to approximately $11.5 \mathrm{~m} \mathrm{~s}^{-1}$ and a secondary positive potential temperature anomaly emerges at $z=3 \mathrm{~km}$ (Figs. $4 \mathrm{e}, \mathrm{f})$. During this time the upper-tropospheric $(6<z<$ $9 \mathrm{~km}$ ) tangential winds have also been accelerated. Although the surface pressure on the system scale undergoes only a small deepening of $\approx 2 \mathrm{mb}$ (not shown), the tangential wind undergoes a rather significant structural transformation.

Figure 5 shows the azimuthal mean transverse circulation and mean diabatic heating rate on the innermost domain at $t=24 \mathrm{~h}$. It is evident that a system-scale mean radial inflow has developed at midlevels and near the surface. The mean near-surface $(0<z<2 \mathrm{~km})$ inflow is approximately $1 \mathrm{~m} \mathrm{~s}^{-1}$, the inflow in the midtroposphere exceeds $3 \mathrm{~m} \mathrm{~s}^{-1}$, and the uppertropospheric radial outflow is approximately $7 \mathrm{~m} \mathrm{~s}^{-1}$. The mean vertical velocity ranges from $0.4 \mathrm{~m} \mathrm{~s}^{-1}$ at low levels to a maximum of $1.4 \mathrm{~m} \mathrm{~s}^{-1}$ at midlevels, with the strongest mean ascent located near $50-\mathrm{km}$ radius from the initial MCV center (Fig. 5c). It is evident that a well-defined mean toroidal (secondary) circulation has developed by $24 \mathrm{~h}$.

The diabatic heating rate $\theta$ is generally dominated by the latent heat release in the convective updrafts. The azimuthally averaged diabatic heating rate $\overline{\dot{\theta}}$ often exceeds $18 \mathrm{~K} \mathrm{~h}^{-1}$ in the mean ascending regions (e.g., Fig. $5 \mathrm{~d})$. The near colocation of positive diabatic heating maxima and upward vertical velocity maxima is a persistent feature of the control and sensitivity experiments. The coarse-grained latent heating associated with the ensemble of VHTs is the dominant forcing term in the Sawyer-Eliassen equation that will be shown in section 7 to approximately describe the system-scale toroidal circulation.

By the end of the control simulation $(t=72 \mathrm{~h})$, the vortex has surpassed the threshold of hurricane intensity. The symmetric vortex at this time is highlighted in Fig. 6. The simulated vortex resembles a mature hurricane with maximum mean tangential winds of approximately $46 \mathrm{~m} \mathrm{~s}^{-1}$. The vortex exhibits all of the characteristics expected of a mature hurricane, including a tilted eyewall, a vigorous in-up-and-out mean toroidal circulation with a shallow inflow near the surface, and weak mean subsidence in the eye region (Palmen and Newton 1969; Anthes 1982; Jorgensen 1984). Vigorous diabatic heating is evident in the eyewall region, with maximum azimuthal mean heating values now exceeding $80 \mathrm{~K} \mathrm{~h}^{-1}$. The RMW has contracted from approximately $50 \mathrm{~km}$ at $t=24 \mathrm{~h}$ (Fig. $5 \mathrm{~b}$ ) to approximately 10 $\mathrm{km}$ at $t=72 \mathrm{~h}$.

For the experiments in Table 1 that evolve into a hurricane, the simulated eyes are found to equilibrate to a roughly similar horizontal dimension. Since there is no asymmetric environmental forcing to "smear out" the vorticity (PV), and hence broaden and weaken the tangential winds, the simulated storms qualitatively resemble "midget" typhoons observed in the western Pacific basin (e.g., Lander 1994).

\section{b. Results for sensitivity experiments}

The sensitivity experiments are grouped into five categories: (a) horizontal resolution, (b) convective and thermodynamic processes, (c) perturbations in initial MCV structure, (d) absence of latent/sensible heat fluxes or momentum fluxes at ocean surface, and (e) absence of Coriolis parameter. Table 1 highlights pertinent details and variations observed in each experiment. The outcome of the sensitivity experiments is summarized below:

(a) Parameters are kept the same as experiment A1, except the horizontal resolution is decreased somewhat (expt A2: $\Delta x=\Delta y=3 \mathrm{~km}$ ). The VHT phenomenology and "bottom-up" development is similar to experiment A1. A surface-concentrated tropical depression strength vortex forms at approximately $24 \mathrm{~h}$, and hurricane intensity is attained by $72 \mathrm{~h}$.

(b) The warm bubble method employed in experiments $\mathrm{A} 1$ and $\mathrm{A} 2$ is a standard expedient for stimulating convection (see footnote 4 for more detail). The main results are found to be independent of the bubble initialization scheme. For example, in experiment B1 the same MCV plus Jordan sounding was initialized without a warm bubble. After approximately $14 \mathrm{~h}$, deep convection erupted on the edge of the initial MCV in a ring centered near the radius of maximum surface tangential velocity where the positive Ekman pumping velocity is maximal (Eliassen and Lystad 1977; Montgomery et al. 2001). The convective ring soon broke down into asymmetric concentrations of VHTs. The ensuing VHT phenomenology looks indistinguishable from the control experiment, one day earlier. A tropical depression strength surface vortex forms by $48 \mathrm{~h}$; tropical storm intensity is attained by $72 \mathrm{~h}$. 

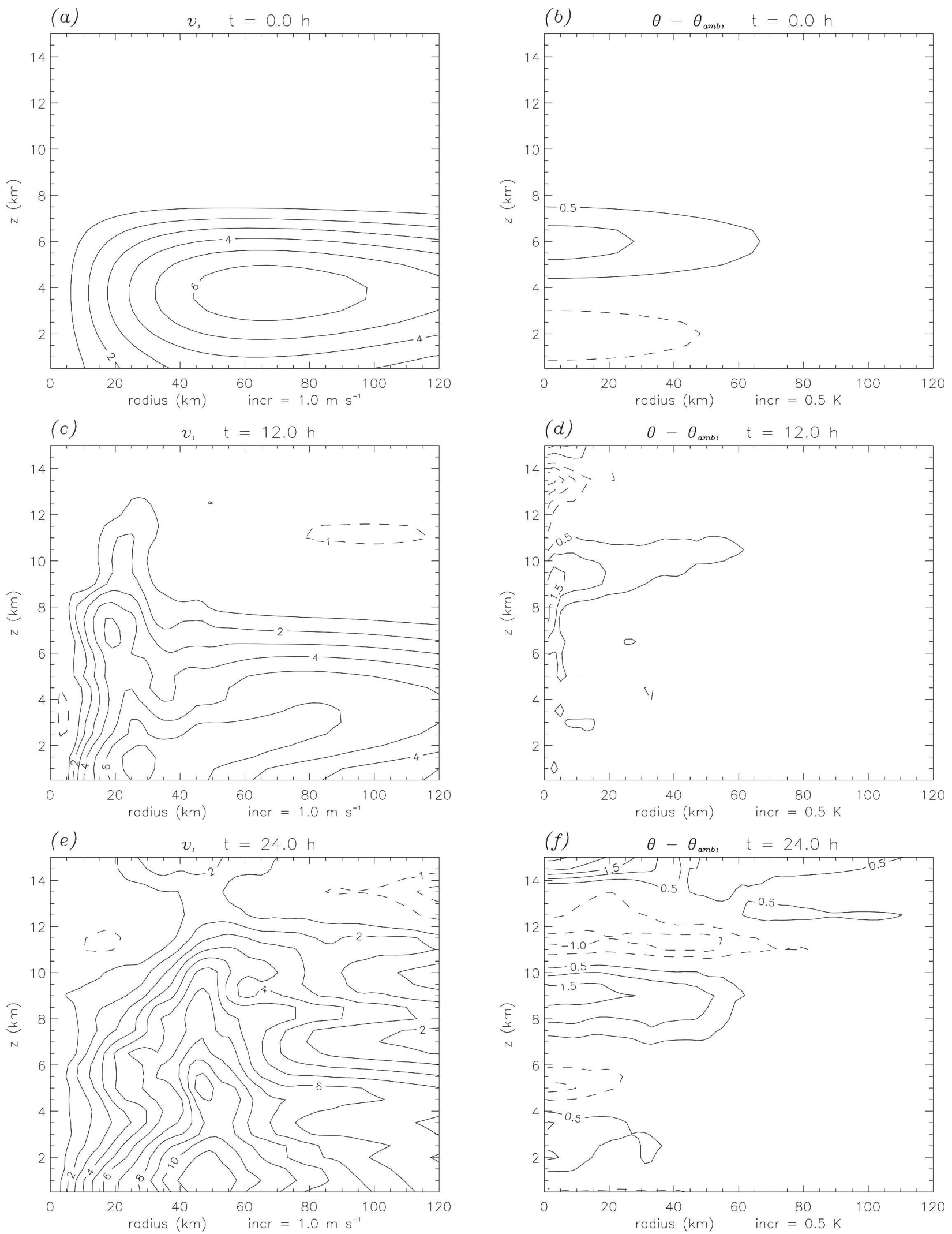

FIG. 4. Azimuthal average of tangential velocity and departure of potential temperature from ambient at (a), (b) $t=0$, (c), (d) 12 , and (e), (f) $24 \mathrm{~h}$ into the control simulation, expt A1. The zero contour is omitted. Negative contours are dashed. Note the transition from cold core to warm core in the lower troposphere. 

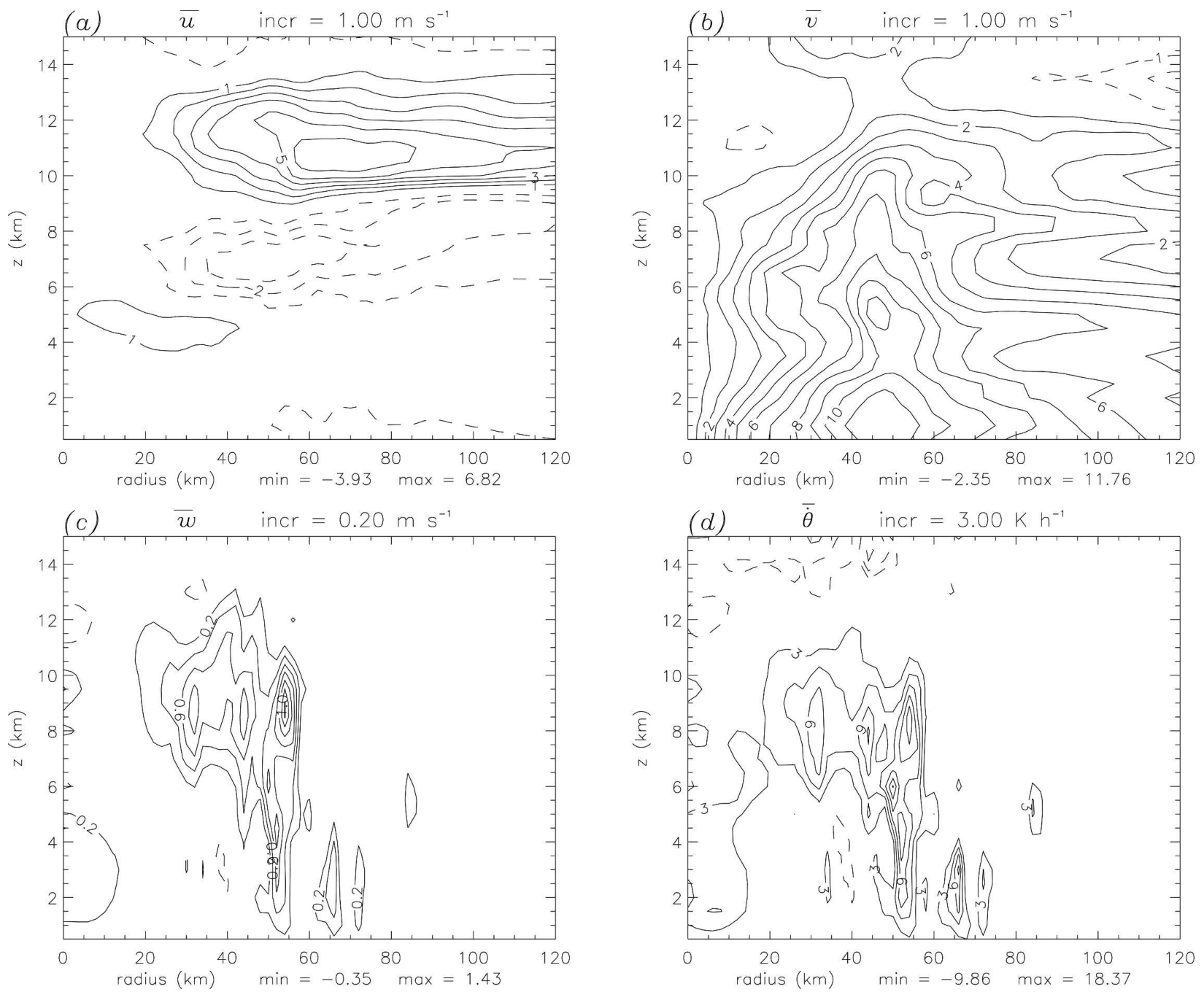

FIG. 5. Azimuthal averages of (a) radial wind, (b) tangential wind, (c) vertical velocity, and (d) diabatic heating rate at $t=24 \mathrm{~h}$ from the control simulation, expt A1. The zero contour is omitted. Negative contours are dashed.

The bottom-up development observed in experiment A1 is broadly unchanged by reductions in CAPE (expts B2, B3, and B4), reductions in SST (expts B5 and B6), variations in the horizontal placement of the initial warm bubble (expt B7), variation in the spatial extent of the initial moisture anomaly (expt B8), or variations in the initialization of the bubble in relation to the diurnal cycle (expt B9). See Table 1 for details.

(c) Perturbations in initial vortex size, intensity, and height above sea level (expts C2, C3, C4, and C5) still yield a bottom-up development similar to experiment A1. A somewhat longer time interval is required, however, in order to spin up a tropical depression-strength vortex for the cases of a weak $\left(5 \mathrm{~m} \mathrm{~s}^{-1}\right.$ ) initial MCV (expts $\mathrm{C} 3$ and $\mathrm{C} 4$ ) or a slightly elevated MCV (maximum tangential winds at $z=5 \mathrm{~km}$, expt C5). An exception is experiment $\mathrm{C} 1$, in which the initial MCV is totally absent. No intense VHTs are generated, and no surface spinup is observed whatsoever without an initial MCV.

(d) In the absence of latent/sensible heat, or momentum, fluxes at the ocean surface, a tropical depression-strength surface vortex still develops (see, e.g., expts D1 and D2). However, no further development is predicted by the cloud model for expt D1. See section 4 for further discussion of experiment D1.

(e) In the absence of planetary vorticity (zero Coriolis) a tropical depression-strength surface vortex still develops by approximately $t=24 \mathrm{~h}$ (expt E1). At later times, however, the surface vortex loses its integrity and weakens in intensity; the convection becomes highly disorganized and asymmetric. 

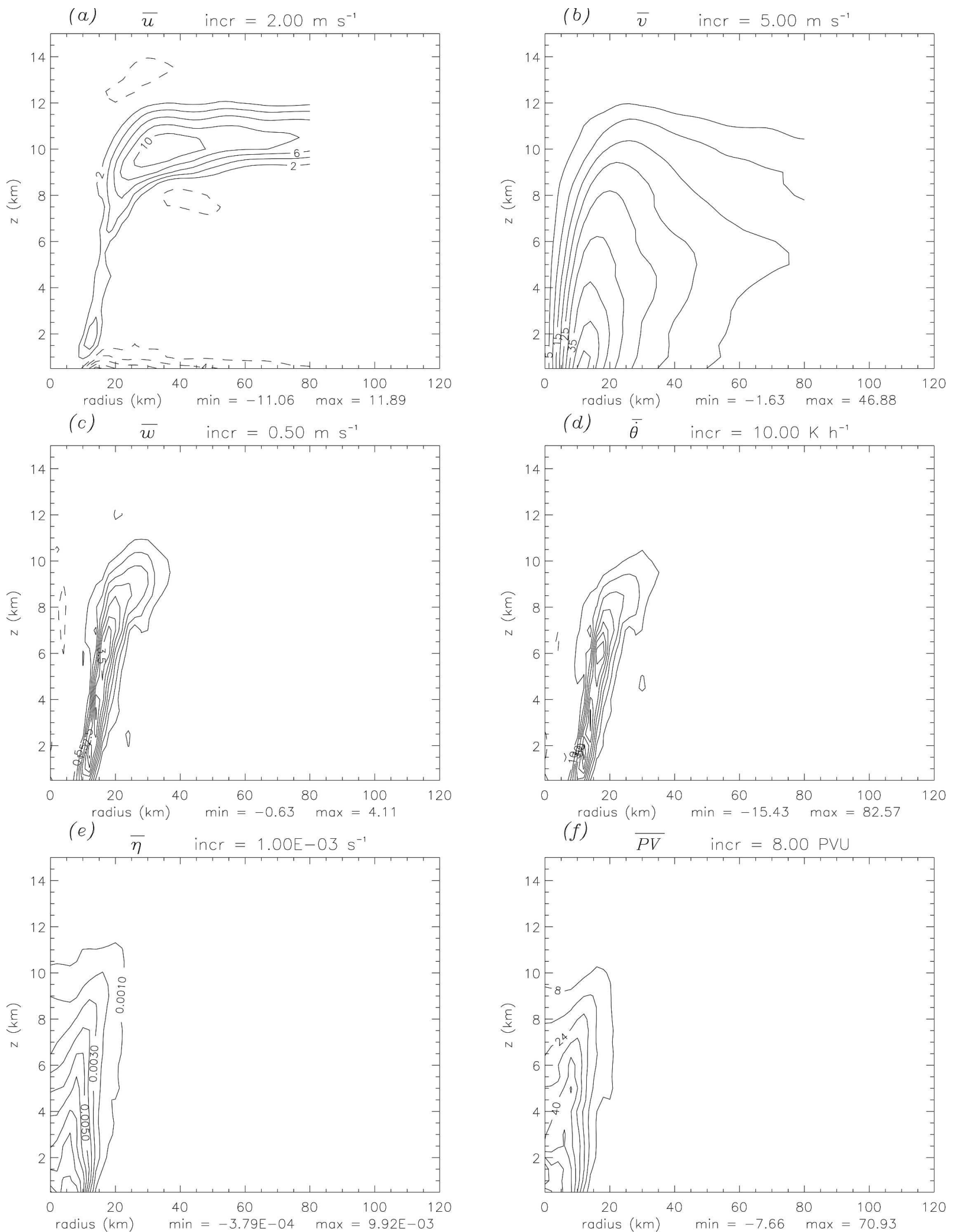

FIG. 6. Azimuthal averages of (a) radial wind, (b) tangential wind, (c) vertical velocity, (d) diabatic heating, (e) absolute vertical vorticity, and (f) Rossby-Ertel PV (in PVU) at $t=72 \mathrm{~h}$ in the control simulation, expt A1. The zero contour is omitted. Negative contours are dashed. 


\section{Some thermodynamic aspects of metamorphosis}

The preceding examples demonstrate that in a conditionally unstable environment, an elevated cyclonic MCV with a weak surface circulation can undergo a transformation to a warm core surface-concentrated tropical depression vortex on meteorologically realistic time scales, depending on flow parameters. Here we examine some thermodynamic aspects of the metamorphosis process.

Although sea-to-air moisture fluxes have been assumed in generating the initial moisture anomaly and CAPE within the initial MCV region, it is of interest to determine whether the surface wind amplification during metamorphosis is also dependent on sea-to-air latent and sensible heat fluxes. Previous work on tropical cyclone intensification has demonstrated that in a convectively neutral environment, enhanced surface fluxes associated with sufficiently strong surface winds can increase the subcloud layer entropy and the core temperature field (Rotunno and Emanuel 1987). The result is a positive feedback to the warm core cyclone, and the system-scale vortex amplifies. This positive feedback process is called wind-induced surface heat exchange amplification.

Recall the sensitivity experiment performed with seato-air latent and sensible heat fluxes turned off (expt. D1, see Table 1). As in the control case, experiment D1 exhibits the emergence of a population of VHTs within the cyclonic MCV and a similar metamorphosis on the system scale (not shown). Given the absence of sea-toair heat fluxes during this experiment, one does not expect further intensification of the seedling surface vortex (Malkus and Riehl 1960; Rotunno and Emanuel 1987). Indeed, once the surface tangential winds reach approximately $12 \mathrm{~m} \mathrm{~s}^{-1}$, no further amplification is predicted by the cloud model. The maximum surface winds remain at approximately $12 \mathrm{~m} \mathrm{~s}^{-1}$ through $72 \mathrm{~h}$. Based on these experiments we conclude that MCV metamorphosis in an initially unstable environment is not critically dependent on WISHE amplification.

Further insight into the metamorphosis process may be obtained on examining the evolution of $\theta_{e}$ for the control experiment at the lowest atmospheric grid level $(z=197 \mathrm{~m})$. Figure 7 shows $\theta_{e}$ (using Bolton 1980) at $t=3,15,24$, and $39 \mathrm{~h}$. The surface $\theta_{e}$ ranges from a minimum near $340 \mathrm{~K}$ (white and light blue) to a maximum of $360 \mathrm{~K}$ (purple). At $t=0 \mathrm{~h}$, the $\theta_{e}$ maximum $(\approx 356 \mathrm{~K})$ resides at the domain center, due principally to the initially enhanced low-level moisture distribution near the center of the model domain. Between $t=0$ and $t=15 \mathrm{~h}$ the initial $\theta_{e}$ maximum in the central region has been eroded by the action of convective down- drafts, which act to transport (lower) $\theta_{e}$ from midtropospheric levels $(3<z<5 \mathrm{~km})$ to the surface. By $t=$ $24 \mathrm{~h}, \theta_{e}$ exhibits a quasi-spiral structure of reduced values $(\approx 342-346 \mathrm{~K})$ that encircle the central region near $r=50 \mathrm{~km}$; a maximum of $\theta_{e}(\sim 354-356)$ has reemerged near the center of the system-scale circulation. By $t=$ $39 \mathrm{~h}$, areas of strongly depressed $\theta_{e}$ have been almost eliminated; the central region now exhibits a welldefined maximum, with values ranging from $\sim 354$ to $360 \mathrm{~K}$, the largest of which exceed the initial surface $\theta_{e}$ maximum.

The existence of convective downdrafts and depressed $\theta_{e}$ in the incipient formation region raises the question of how the system-scale development is able to proceed. One clue may be obtained by extracting thermodynamic soundings in the vicinity of VHT events suggested by Fig. 7. In regions of strongly depressed surface $\theta_{e}$ (e.g., sounding A of Fig. 8a, labeled A in Fig. 7b, at $x=-46 \mathrm{~km}, y=14 \mathrm{~km}$ ), the lower troposphere has been dried; the near-surface layer has been cooled; and the overlying air has been warmed and stabilized. Soundings within these strongly depressed $\theta_{e}$ regions resemble onion soundings that are often associated with downdrafts in subsaturated air (Zipser 1977; Fig. 5 of Harr and Elsberry 1996; Fig. 5 of Harr et al. 1996). On the periphery of these $\theta_{e}$ cold pools, however, the surface temperature and moisture are found to be less strongly depressed. For example, consider the two soundings in Figs. 8b,c at $t=24 \mathrm{~h}$ (labeled B and C in Fig. 7c). Sounding B is located at $x=-72 \mathrm{~km}$ and $y=22 \mathrm{~km}$; sounding $\mathrm{C}$ is located at $x=-34 \mathrm{~km}$ and $y=50 \mathrm{~km}$. Although the near-surface air at $\mathrm{C}$ is found to possess a slightly larger surface dewpoint depression than the initial air at similar radius (not shown), the near-surface air at $\mathrm{C}$ is still conditionally unstable. Thus, despite the detrimental downdrafts, air on the periphery of the $\theta_{e}$ deficit regions is still able to support deep convection triggered by the spreading gust front boundaries (Zipser and Gautier 1978). These boundaries are confined laterally due to the increased rotation and the convergent near-surface inflow generated by the aggregate of VHTs (see section 7 for more detail).

The three soundings shown in Fig. 8 also exhibit significant moistening of the middle and upper troposphere relative to that of the initial environment (cf. Fig. 1). The only process that can accomplish this moistening on these time scales is the vertical moisture transport associated with the VHTs. Therefore, despite the detrimental action of VHT-induced downdrafts that create local $\theta_{e}$ cold pools near the surface, the VHTs contribute positively to humidifying the middle and upper troposphere. The thermodynamics of the simulated 

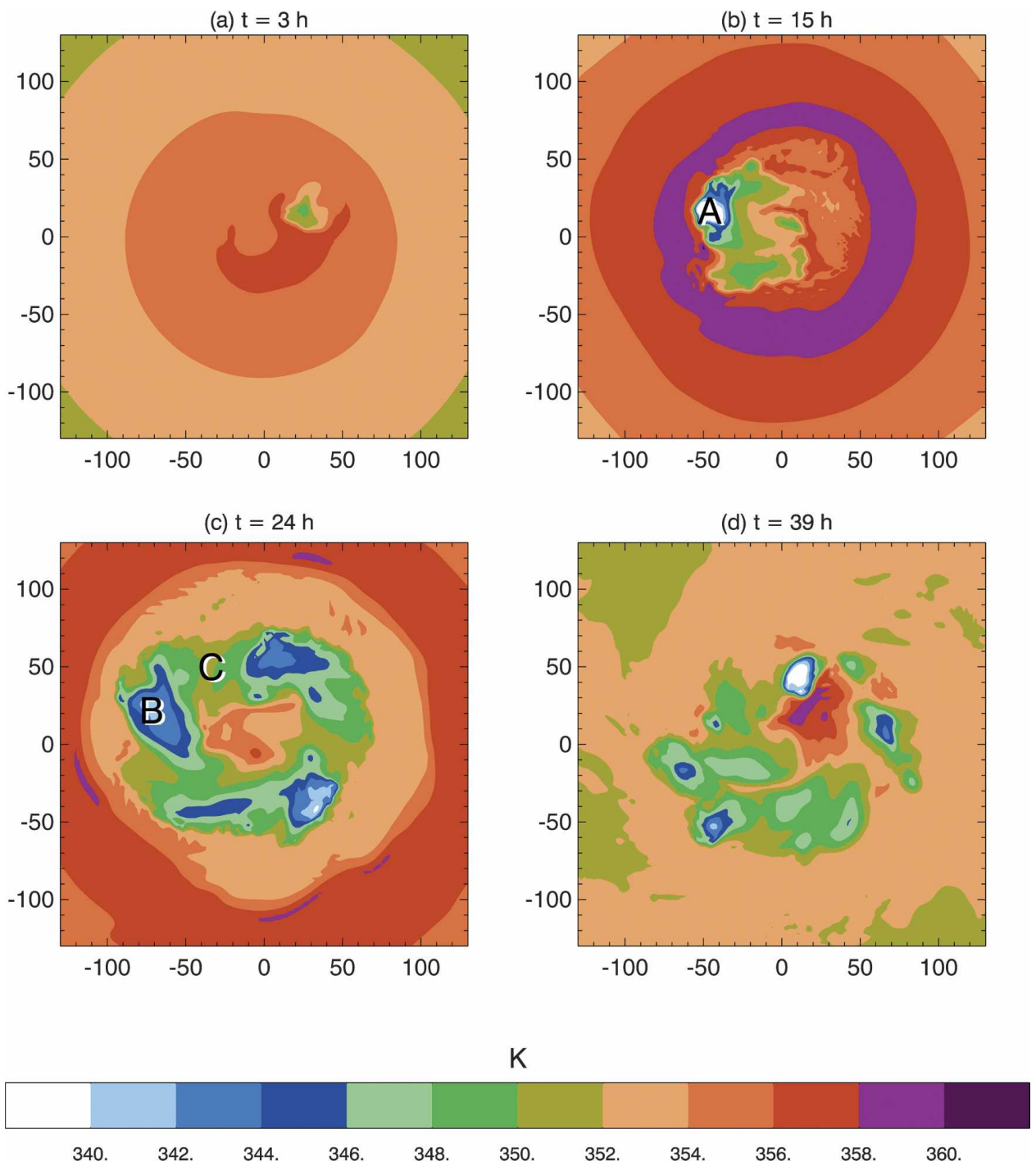

Fig. 7. Simulated $\theta_{e}$ near lower surface $(z=197 \mathrm{~m}$ ) at (a) $t=3$, (b) $t=15$, (c) $t=24$, and (d) $t=39$ $\mathrm{h}$ from control simulation, expt A1. Axes are in $\mathrm{km}$; contour interval is $2 \mathrm{~K}$. The locations marked A, B, and $\mathrm{C}$ are marked in (b) and (c) to indicate the locations of vertical soundings shown in Fig. 8.

metamorphosis evidently involves an interplay between two competing factors: conditional instability of the local environment and VHT-induced column moistening and local near-surface cooling and drying on the periphery of VHT events.

\section{VHT phenomenology}

Although a universally accepted definition of a convective hot tower does not exist, we follow common practice and define a hot tower as an intense convective tower that reaches and/or penetrates the model tropopause $(z \approx 15 \mathrm{~km})$. Elaborating on this definition, $\mathrm{J}$. Simpson referred to hot towers as "undilute or nearly undilute penetrative cumulonimbus clouds that carried high $\theta_{e}$ air from the subcloud layer to the upper troposphere" (Simpson et al. 1998; Zipser 2003; Anthes 2003). When an intense updraft forms in a vorticity-rich environment, however, another factor must be considered. As demonstrated by H04, convective hot towers forming in a vorticity-rich local environment may be highly vortical in nature, possessing intense vertical vorticity within or near their cores. But how are the low-level vertical vorticity anomalies produced?

\section{a. VHT structure and kinematics}

Having established that the spinup process is similar among the majority of sensitivity experiments, we will 

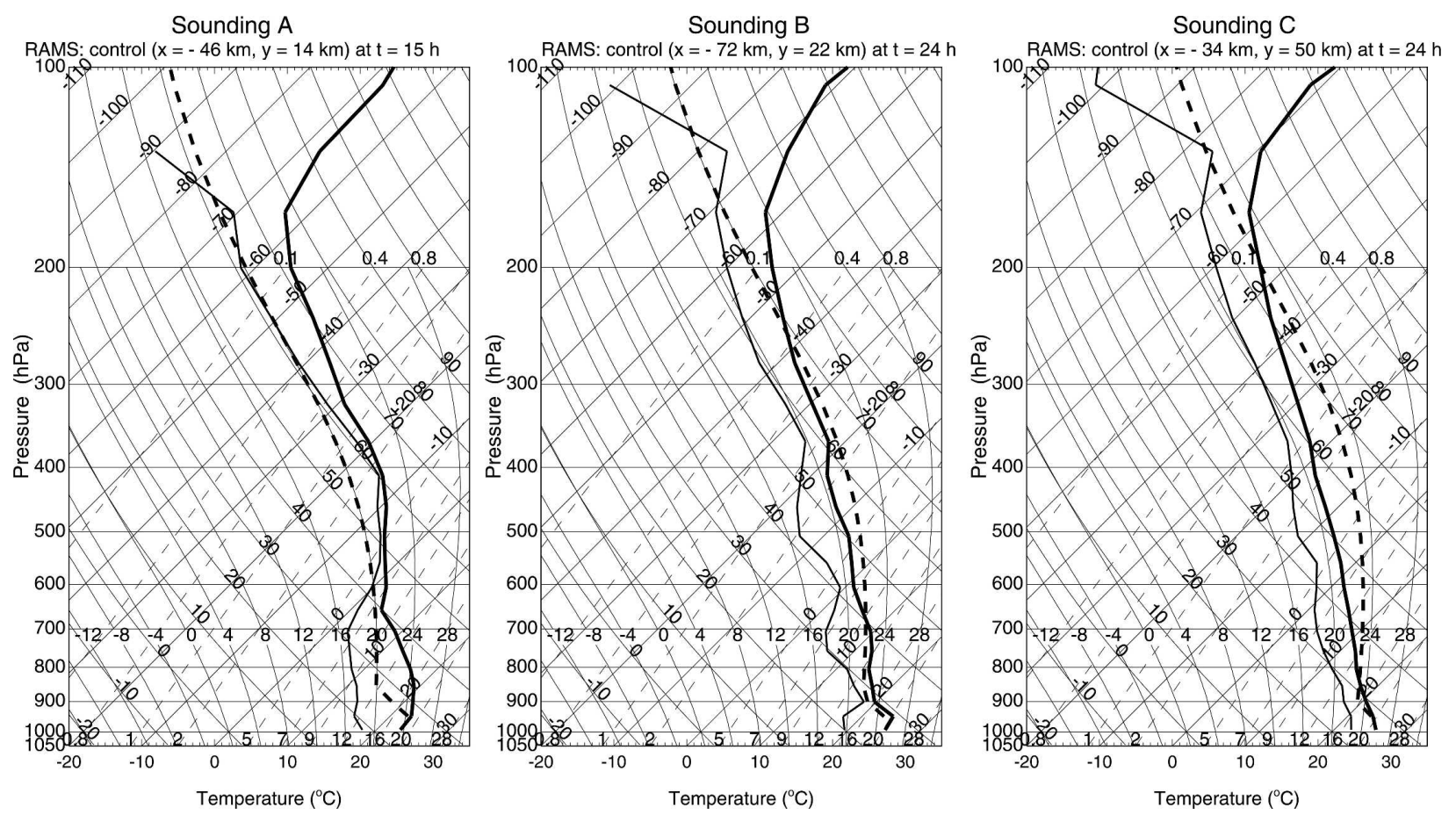

FIG. 8. Skew $T-\log p$ vertical soundings of locations A, B, and C shown in Fig. 7. The temperature sounding is the thick solid line, dewpoint temperature is the thin solid line, and lifted parcel ascent path is the dashed line.

use experiment A1 to examine the convective/vorticity dynamics of the VHTs. In the control case (expt A1), the model-generated updrafts range from 5 to $20 \mathrm{~km}$ in diameter. For the purpose of this study, we define a model hot tower as an updraft with a vertical velocity signature of greater than or equal to $1 \mathrm{~m} \mathrm{~s}^{-1}$ extending from $z=1 \mathrm{~km}$ to at least $15 \mathrm{~km}$, the approximate level of the model tropopause. Maximum instantaneous vertical velocities in the range of $20-35 \mathrm{~m} \mathrm{~s}^{-1}$ are observed at height levels of 9-12 km within hot tower cores. An azimuthally averaged composite VHT has been constructed based on the first $24 \mathrm{~h}$ of model data. The composite VHT has a maximum mean vertical velocity of $15 \mathrm{~m} \mathrm{~s}^{-1}$ at $z=9 \mathrm{~km}$ (not shown). The average lifespan of these VHT structures is on the order of one hour, although individual towers have been tracked for as long as $3 \mathrm{~h}$. Many updrafts, but by no means all, are observed to achieve our definition of a hot tower for brief time periods during their life cycle, and sometimes reach hot tower status multiple times.

The hot towers have substantial anomalous vorticity values near their cores. To illustrate this, we show the absolute vertical vorticity associated with the first hot tower to form in the control experiment in Fig. 9. The first updraft forms at low levels approximately $20 \mathrm{~min}$ into the simulation (Fig. 9a). It achieves hot tower status at $t=40 \mathrm{~min}$, extending vertically to $z=16 \mathrm{~km}$ and with an instantaneous maximum vertical velocity of $33 \mathrm{~m} \mathrm{~s}^{-1}$ at $z=10 \mathrm{~km}$ (not shown). The lifetime of this initial updraft spans roughly $2 \mathrm{~h}$. As Fig. $9 \mathrm{~b}$ shows, a dipole of anomalous absolute vertical vorticity is generated by the initial updraft. A similar dipole structure is evident in dry Rossby-Ertel PV (not shown). As this updraft attains hot tower status at $40 \mathrm{~min}$, the positive portion of the vorticity dipole structure attains values on the order of $10^{-3} \mathrm{~s}^{-1}$ within the model troposphere. This anomalous cyclonic vorticity is significantly larger than that of the local environment just prior to the formation of this updraft. For instance, at $z=9 \mathrm{~km}$ the vertical vorticity at $t=0 \mathrm{~min}$ is $3.8 \times$ $10^{-5} \mathrm{~s}^{-1}$ in the vicinity of the initial updraft. Forty minutes later, the convectively generated vorticity maximum at that level is $4.8 \times 10^{-3} \mathrm{~s}^{-1}$, more than 100 times the preconvective value. In general, the VHT-related vorticity anomalies range from 3 to 100 times larger than the vorticity of their local environment.

VHTs are generally not advected with the local flow as passive scalars. The updraft cores associated with the VHTs are found to rotate about the MCV at approximately $0.6-0.9$ times the local azimuthal mean tangential velocity. This phenomenon has also been observed for eyewall mesovortices in the $1.3-\mathrm{km}$ horizontal grid spacing simulation of Hurricane Bob (1991; Fulton 
(a) $\mathrm{t}=20 \mathrm{~min}$.
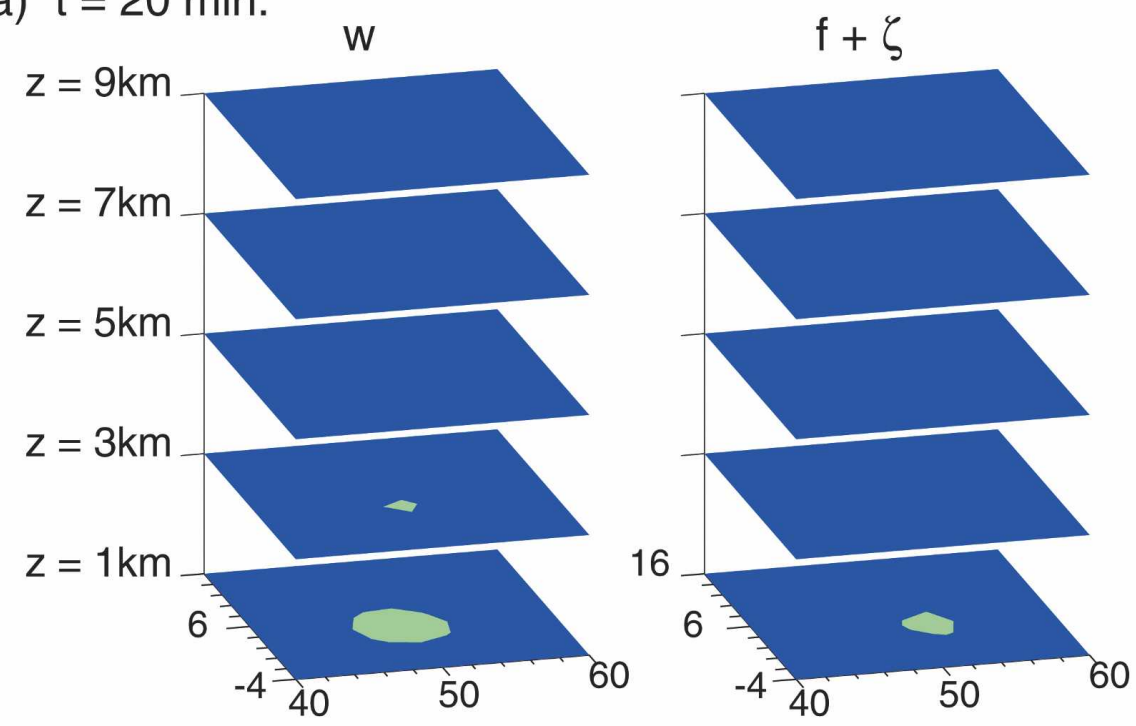

(b) $\mathrm{t}=40 \mathrm{~min}$.

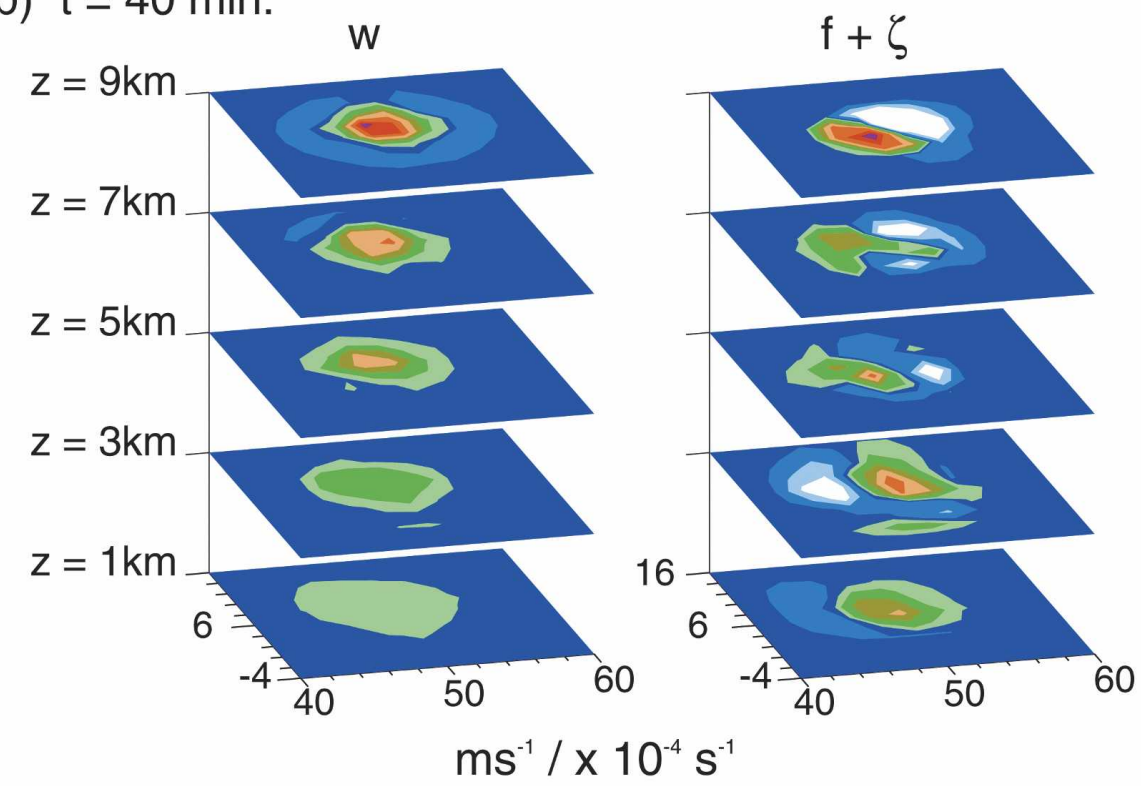

$\begin{array}{llllllllllll}-5 /-10 & -3 /-5 & -1 / 0 & 1 / 2.5 & 5 / 5 & 10 / 10 & 15 / 15 & 20 / 20 & 25 / 30 & 30 / 40 & 35 / 50\end{array}$

FIG. 9. Example vertical velocity $w\left(\mathrm{~m} \mathrm{~s}^{-1}\right)$ and absolute vertical vorticity $\eta\left(\times 10^{-4} \mathrm{~s}^{-1}\right)$ signatures associated with deep cumulus convection in control simulation, expt A1, at (a) $t=20$ min and (b) $t=$ $40 \mathrm{~min}$. Horizontal cross sections are $20 \mathrm{~km} \times 20 \mathrm{~km}$ subdomains centered at (a) $x=50 \mathrm{~km}, y=4 \mathrm{~km}$, and (b) $x=50 \mathrm{~km}, y=6 \mathrm{~km}$. The existence of a strong vorticity dipole collocated with the core of the hot tower and its orientation suggest tilting of ambient vorticity associated with the initial MCV.

2001, based on the simulation by Braun (2002) and the 2.0-km horizontal grid spacing simulation of Hurricane Bonnie (1998; Braun et al. 2006). These findings are consistent with the idea that the vorticity towers in the high vorticity region of the seedling storm, and the intense updrafts that support them, behave in some respects like Rossby solitons (Nezlin and Snezhkin 1993; McWilliams et al. 2003). 
(a)

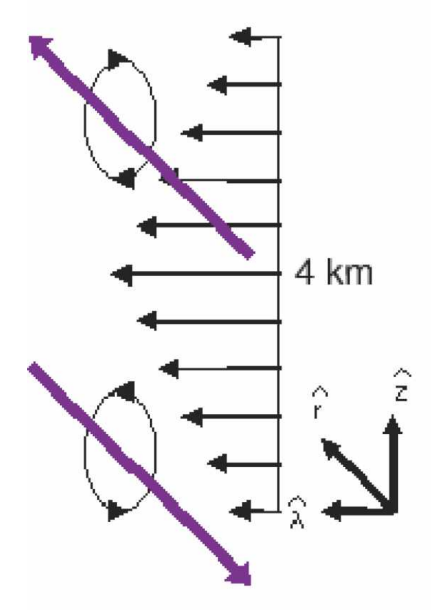

(b)
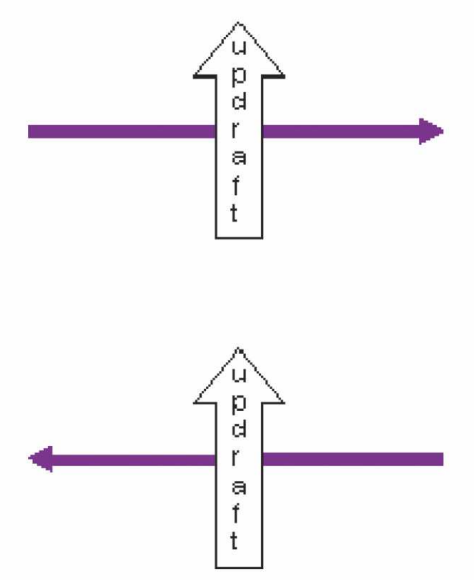

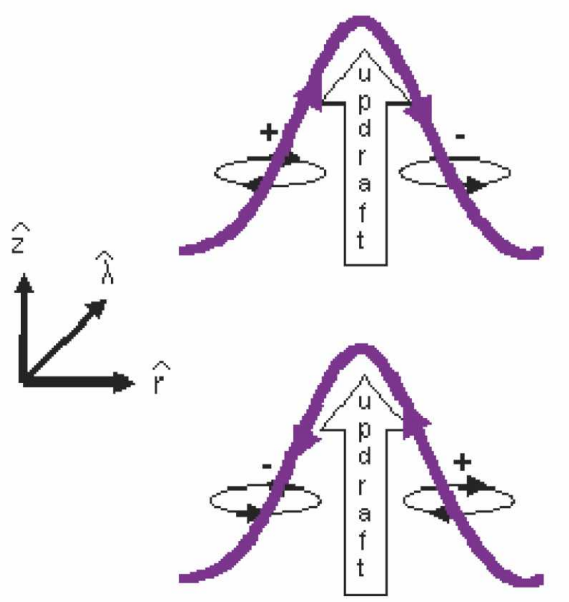

FIG. 10. Schematic of vortex tilting within the initial MCV. Purple lines represent vortex filaments. (a) Radial vorticity generated by vertical shear profile of initial MCV. (b) Updraft tilts radial vortex filament upward, generating a vertical vorticity dipole with negative relative vorticity radially inward (outward) at heights below (above) $z=4 \mathrm{~km}$.

\section{b. Vortex tilting and stretching}

Given that the dipolar structure of the vorticity anomalies is approximately colocated with the hot towers, the following vorticity generation mechanism is proposed: The initial MCV provides an environment rich in horizontal and vertical vorticity. As the first updraft forms, it tilts ambient horizontal vorticity into the vertical while at the same time stretching MCVgenerated vertical vorticity. As the updraft intensifies to become a hot tower, both ambient and tiltinggenerated vertical vorticity is stretched even more, leading to a strong convectively generated vertical vorticity anomaly. At later times in the simulation(s) the convergence/stretching of near surface $(0<z<2 \mathrm{~km})$ vorticity by the convective plumes dominates the generation of vorticity by tilting processes (not shown).

A rudimentary understanding of the cloud-scale dynamics at early times can be obtained by examining the evolution of the updrafts. The local and system-scale dynamics at later times are analyzed in sequel. As described previously, our control simulations are initialized with a weak midlevel vortex elevated above the sea surface. At radii less than $75 \mathrm{~km}$, the main vortex has a basic-state cyclonic tangential velocity field that increases in magnitude with height below $z=4 \mathrm{~km}$ and decreasing above. Ignoring buoyant effects for the time being, we can consider the horizontal vorticity profile at the initial time as being due solely to the vertical wind shear of the initial MCV. As sketched in Fig. 10, this vertical shear profile will generate a radial vorticity pro- file that, when tilted upward by an updraft, generates negative (positive) relative vertical vorticity anomalies on the radially inward side of the updraft below (above) $z=4 \mathrm{~km}$. As positive vertical vorticity is generated in the region of an updraft, vortex tube stretching further intensifies the positive vorticity anomaly. Returning to Fig. 9b, we can see that the VHT's vorticity dipole structure exhibits this orientation and magnitude difference, supporting our proposed mechanism. Unlike the tilting term, however, the intensification of vertical vorticity by stretching can be exponential if the convergence is approximately constant during the parcel's ascent through the tower.

The mechanisms of vertical vorticity generation may be clarified on recalling the material form of the vertical vorticity equation:

$$
\begin{aligned}
\frac{D \zeta}{D t}= & -(f+\zeta)\left(\frac{\partial u}{\partial x}+\frac{\partial v}{\partial y}\right)-\left(\frac{\partial w}{\partial x} \frac{\partial v}{\partial z}-\frac{\partial w}{\partial y} \frac{\partial u}{\partial z}\right) \\
& +\frac{1}{\rho^{2}}\left(\frac{\partial \rho}{\partial x} \frac{\partial p}{\partial y}-\frac{\partial \rho}{\partial y} \frac{\partial p}{\partial x}\right)+\frac{\partial F_{y}}{\partial x}-\frac{\partial F_{x}}{\partial y},
\end{aligned}
$$

where $\zeta$ is the relative vertical vorticity. The terms on the right-hand side of this equation represent contributions to the material rate of change of $\zeta$ due to horizontal convergence, tilting, solenoidal effects, and subgrid-scale flux derivatives, respectively. Many cloud models (including RAMS) employ a Boussinesq-like approximation in the virtual potential temperature and neglect horizontal variations of virtual potential tem- 
(a) $t=30 \min$
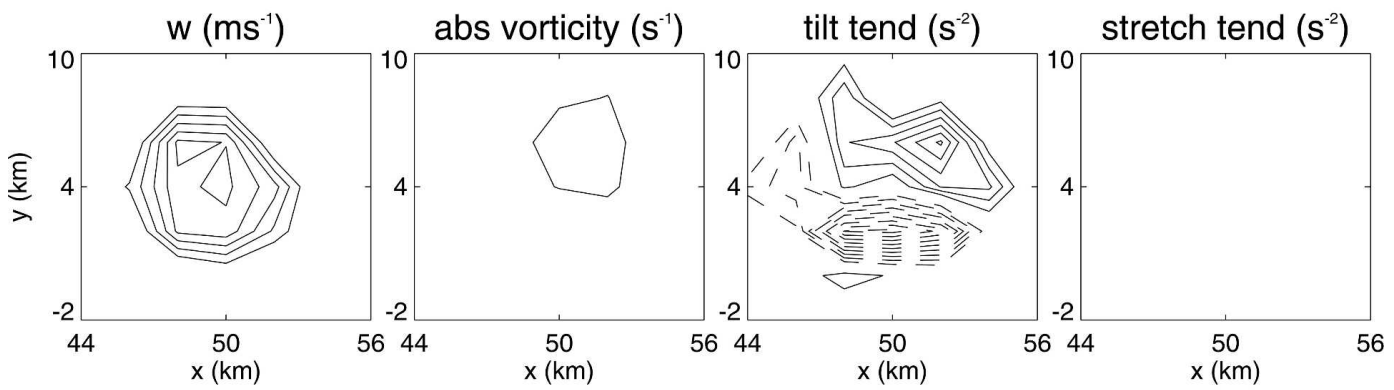

(b) $\mathrm{t}=40 \mathrm{~min}$
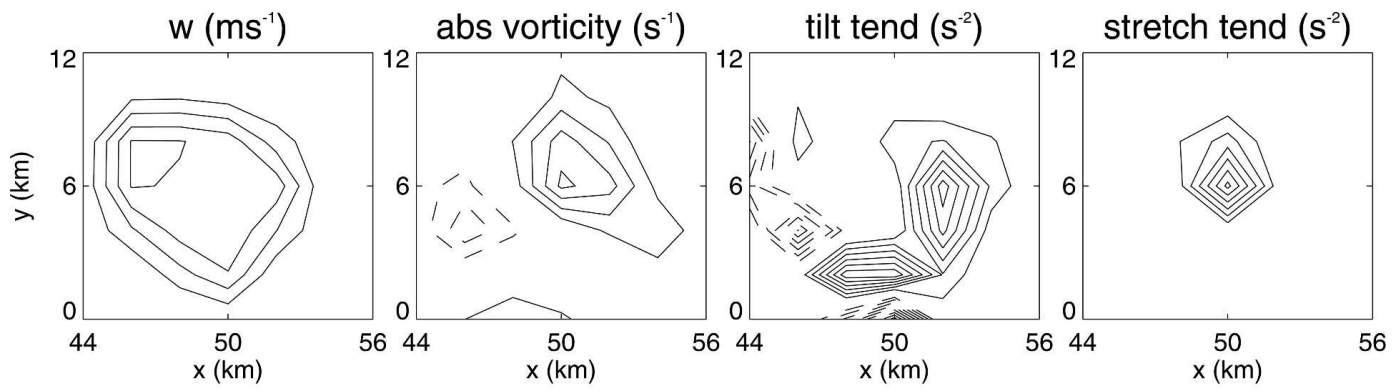

\section{(c) $\mathrm{t}=50 \mathrm{~min}$}
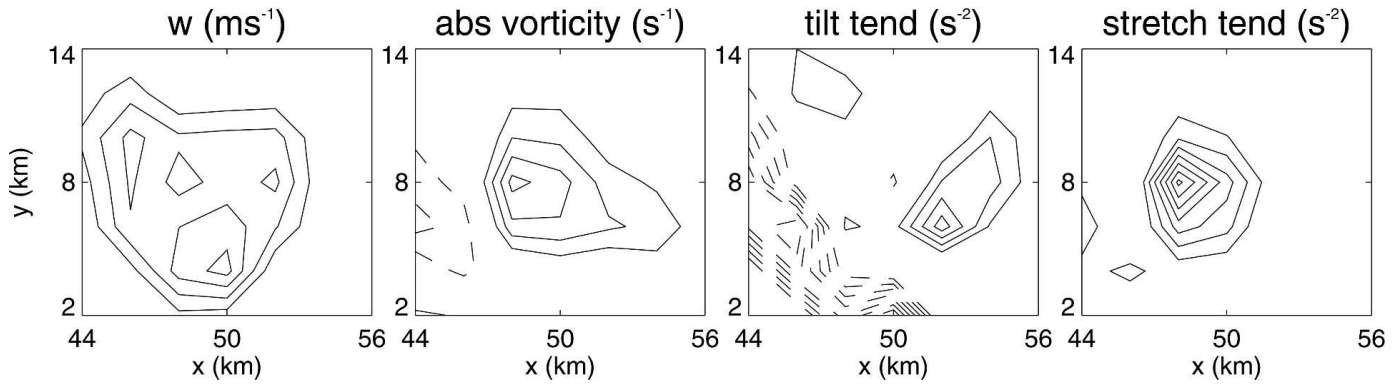

FIG. 11. Model vertical velocity $w$ (contour interval $2 \mathrm{~m} \mathrm{~s}^{-1}$ ) and absolute vertical vorticity $\eta=f+\zeta$ (contour interval $5 \times 10^{-4} \mathrm{~s}^{-1}$ ) for control simulation, expt A1, in conjunction with instantaneous contributions of tilting and stretching tendency terms in equation for material rate of change of vertical vorticity (contour interval $1 \times 10^{-6}$ $\mathrm{s}^{-2}$ ) at (a) $t=30 \mathrm{~min}$, (b) $t=40 \mathrm{~min}$, and (c) $t=50 \mathrm{~min}$. Values displayed on a $20 \mathrm{~km} \times 20 \mathrm{~km}$ horizontal subdomain centered about a vortical hot tower, at height $z=2.5 \mathrm{~km}$. The zero contour is omitted. Negative contours are dashed.

perature in the effective horizontal pressure gradient force. This approximation makes the corresponding third term on the right-hand side of Eq. (5) vanish. The $x$ and $y$ components of the subgrid-scale terms generally yield a small contribution to the net vorticity tendency and will be ignored. ${ }^{5}$ We henceforth focus on the

\footnotetext{
${ }^{5}$ Justification is based on Cram et al. (2002), where an analysis of a RAMS simulation of a convective line with $f=0$ found these subgrid-scale flux terms to be two orders of magnitude smaller than the first two tendency terms on the rhs of Eq. (5).
}

convergence and tilting contributions to the material rate of change of $\zeta$.

As an illustration, let us return again to the first convective hot tower that forms in the control simulation. Following the Eulerian diagnosis outlined in Cram et al. (2002), the tilting and stretching tendencies on the right-hand side of Eq. (5) were computed in the vicinity of this first updraft at various height levels. Since our focus is on vertical vorticity generation at low levels, Fig. 11 shows these tendency values at $z=2.5 \mathrm{~km}$. It is evident that at early stages of this updraft $(t \sim 30 \mathrm{~min})$, most of the vertical vorticity originates from tilting of 
ambient horizontal vorticity into the vertical. As the updraft continues to evolve, however, stretching of cyclonic vertical vorticity starts to dominate the tilting effect. These characteristics are similar to those of rotating convective storms at middle latitudes in weak to moderate vertical shear and moderate CAPE environments (Klemp 1987; Holton 2004, section 9.6).

\section{c. Summary of VHT behavior}

In the presence of modest near-surface vertical vorticity (arising here from a cyclonic MCV), a single convective hot tower can generate a cyclonic vertical vorticity anomaly that is an order of magnitude greater than the ambient vertical vorticity within a time span of $20 \mathrm{~min}$. Although the specific details are likely to be somewhat resolution-dependent until truly cloudresolving scales are attained (e.g., Bryan et al. 2003), and while some of the simulated convective vorticity anomalies are spatially small ( 5-10 km-arguably not well resolved here), it will be shown in upcoming sections that within the dynamically protected environment of the MCV embryo the VHTs tend to persist and interact with each other in ways that serve to strengthen themselves and force a system-scale convergence of near-surface $(0<z<2 \mathrm{~km})$ cyclonic absolute vorticity.

\section{Local and system-scale dynamics}

During the transformation from elevated MCV to tropical depression many diabatic mergers between VHTs are observed. A representative example highlighting a diabatic vortex merger between two distinct VHTs is shown in Fig. 12, which spans a 40-min interval from $t=6 \mathrm{~h}, 40 \mathrm{~min}$ to $t=7 \mathrm{~h}, 20 \mathrm{~min}$ in experiment A1. A three-dimensional snapshot of this merger event (at $t=7 \mathrm{~h}$ ) was already highlighted in Fig. 3. Figure 12 shows a horizontal plan view of dry Rossby-Ertel PV on $z=1 \mathrm{~km}$, and a diagonal cross section A-B through the PV couplet during the highlighted merger event. The magnitude of cyclonic PV at $z=1 \mathrm{~km}$ increases from approximately 18 to $28 \mathrm{PVU}$, where $1 \mathrm{PVU}=$ $10^{-6} \mathrm{~m}^{2} \mathrm{~s}^{-1} \mathrm{~K} \mathrm{~kg}^{-1}$. Once this merger is completed, the maximum near-surface $\mathrm{PV}$ in this region waxes and wanes as other small-scale, discrete PV maxima merge with the "parent" PV region. The cumulative increase of low-level $(0<z<3 \mathrm{~km}) \mathrm{PV}$ accompanying these diabatic merger events is illustrated in Fig. 13. Over the course of $1 \mathrm{~h}, 40 \mathrm{~min}$ the near-surface $(z=500 \mathrm{~m})$ cyclonic PV maximum increases from approximately 12 to 40 PVU, a considerable increase.

\section{a. Local budgets of tangential momentum and potential temperature}

To quantify the role of the discrete, small-scale VHTs and their mergers in the vortex transformation process, local budgets of azimuthal mean tangential momentum and potential temperature have been calculated for particular VHT events, as in H04. The local budgets are carried out in a cylindrical coordinate system. Instead of using a coordinate center that moves with one or the other VHT, we will, for simplicity, calculate local budgets about a fixed event center that is near the vorticity centroid of the merged system. The event center for the case illustrated in Fig. 12, for example, is located at $x=-18 \mathrm{~km}$ and $y=14 \mathrm{~km}$. Diagnoses here are based on traditional budgets for azimuthal-mean tangential momentum (where the sg subscript stands for subgrid)

$$
\frac{\partial \bar{v}}{\partial t}=-\overline{u \eta}-\overline{u^{\prime} \zeta^{\prime}}-\bar{w} \frac{\partial \bar{v}}{\partial z}-\overline{w^{\prime} \frac{\partial v^{\prime}}{\partial z}}+\bar{F}_{\mathrm{sg}}
$$

and azimuthal-mean potential temperature

$$
\frac{\partial \bar{\theta}}{\partial t}=-\bar{u} \frac{\partial \bar{\theta}}{\partial r}-\overline{u^{\prime} \frac{\partial \theta^{\prime}}{\partial r}}-\bar{w} \frac{\partial \bar{\theta}}{\partial z}-\overline{w^{\prime} \frac{\partial \theta^{\prime}}{\partial z}}+\overline{\dot{\theta}}
$$

In Eqs. (6) and (7), the overbar denotes an azimuthal average on constant height levels about the local center; and the prime denotes a departure from the azimuthal mean (eddy). Appendix B provides further details of this diagnostic package. In Eq. (6), the terms on the right-hand side are called the mean radial flux of absolute mean vertical vorticity (MRAV), the eddy radial flux of eddy relative vorticity (ERRV), the mean vertical flux of mean tangential momentum (MVTM), the eddy vertical flux of eddy tangential momentum (EVTM), and, finally, the mean subgrid-scale tendency of tangential momentum, respectively. The terms on the right-hand side of Eq. (7) are similarly called: the mean radial advection of mean potential temperature (MRPT), the eddy radial advection of eddy potential temperature (ERPT), the mean vertical advection of mean potential temperature (MVPT), the eddy vertical advection of eddy potential temperature (EVPT), and the diabatic heating rate (THDOT), respectively.

Figure 14 shows the time-integrated terms (indicated by $<>$ ) in the tangential momentum budget for the highlighted diabatic merger event. The increase in tangential momentum during this event occurs almost entirely within the lowest $4 \mathrm{~km}$ of the model troposphere. All tendency terms contribute to the near-surface 

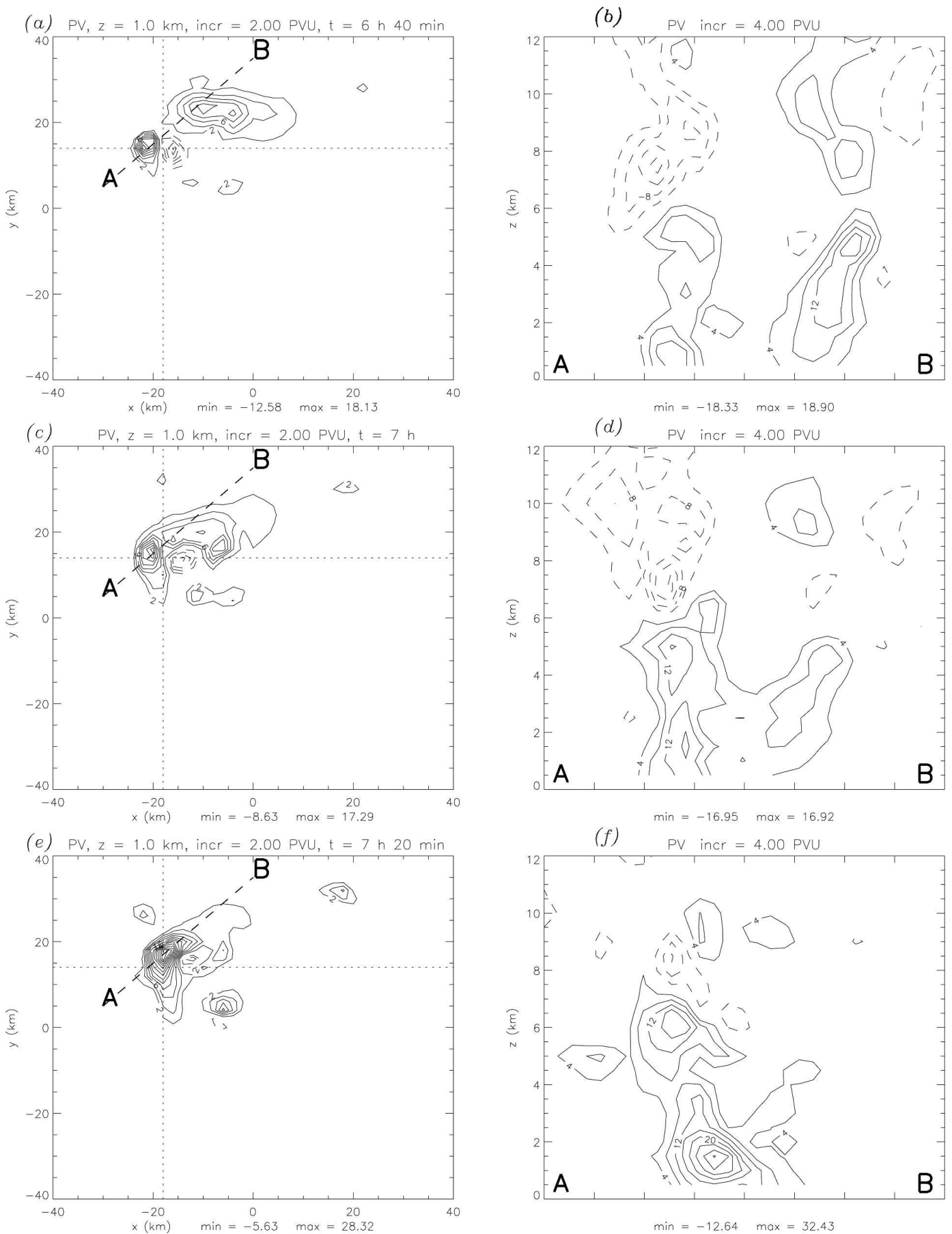

FIG. 12. Rossby-Ertel PV from the control simulation (expt A1) at $z=1 \mathrm{~km}$ at (a) $t=6 \mathrm{~h}, 40 \mathrm{~min}$; (c) $t=7 \mathrm{~h}$; and (e) $t=7 \mathrm{~h}, 20 \mathrm{~min}$. The dashed line connecting points A and B indicates the location of vertical cross sections shown in (b), (d), and (f). Tick marks along the horizontal axis in (b), (d), and (f) are plotted at 5-km intervals. A snapshot during this diabatic merger event at $t=7 \mathrm{~h}$ was highlighted in Fig. 3. The intersection of the thin dotted lines denotes the center location used for the azimuthal averages in the corresponding local tangential momentum and potential temperature budgets for this event (see Figs. 14 and 15, respectively). The zero contour is omitted. Negative contours are dashed. 

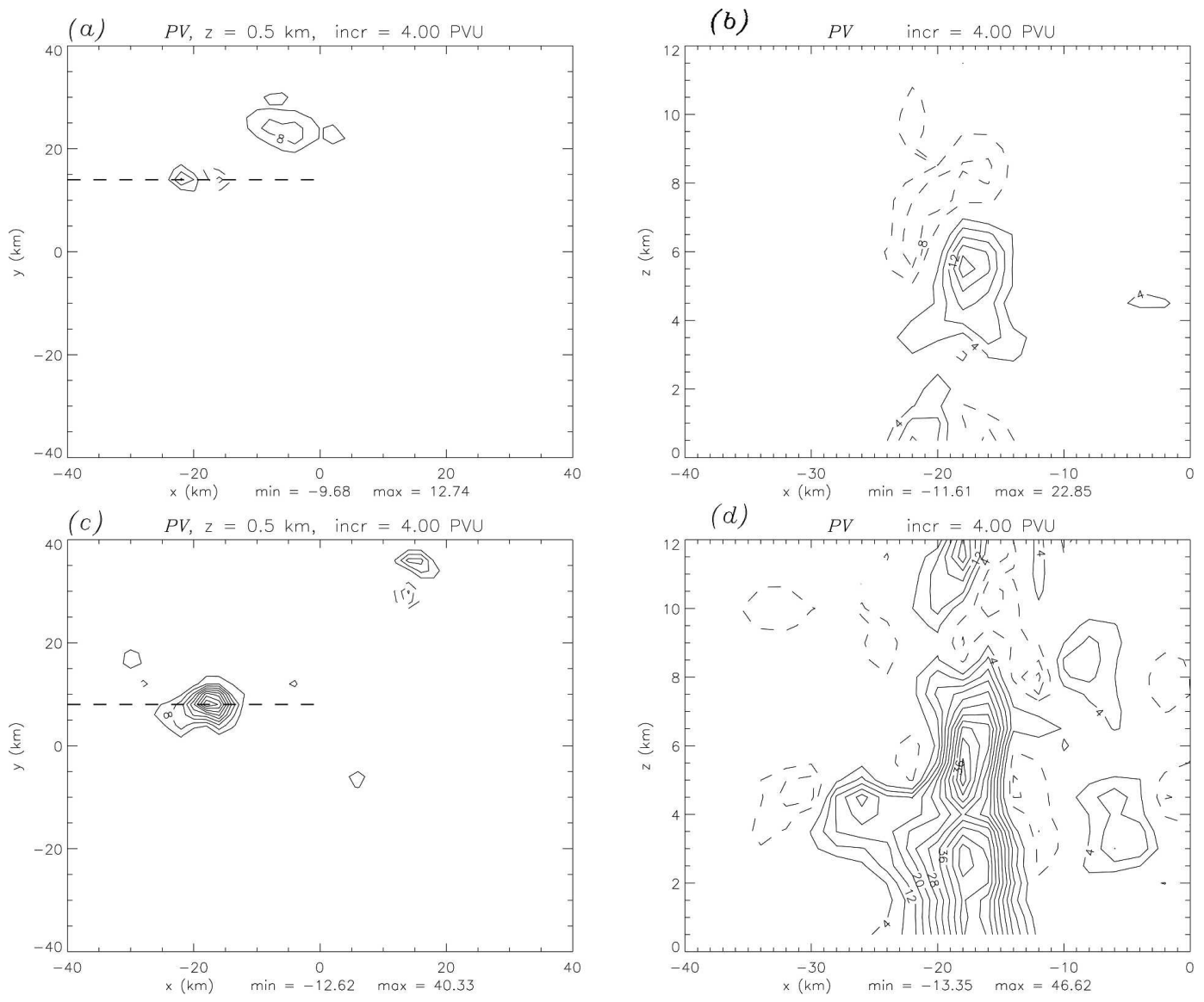

Fig. 13. Rossby-Ertel PV at $z=500 \mathrm{~m}$ at (a) $t=6 \mathrm{~h}, 30 \mathrm{~min}$ and (c) $t=8 \mathrm{~h}, 10 \mathrm{~min}$ from control simulation. expt A1. (b), (d) Vertical cross sections along the dotted lines. The zero contour is omitted. Negative contours are dashed.

spinup except the eddy vertical momentum flux, which is associated with a near-surface spindown maximum of $8 \mathrm{~m} \mathrm{~s}^{-1}$. The mean near-surface tangential velocity increases by approximately $4 \mathrm{~m} \mathrm{~s}^{-1}$ during the merger event (Fig. 14e). This spinup is attributed to the mean radial and mean vertical advection of angular momentum ( $\langle$ MRAV $\rangle,\langle$ MVTM $\rangle)$ (Figs. 14a,c) and also to the radial eddy vorticity flux (〈ERRV $\rangle$ ) (Fig. 14b). The calculated budgets demonstrate that on the convective scale the VHTs contribute positively to a local nearsurface spinup (ME98).

Figure 15 shows the time-integrated tendency terms in the potential temperature equation for the same merger event. The diabatic heating term ( $\langle$ THDOT $\rangle$ ) produces a warming component that exceeds $100 \mathrm{~K}$ over $40 \mathrm{~min}$. This is mostly balanced by the adiabatic cooling term ( $\langle\mathrm{MVPT}\rangle$; Fig. 15c). The near cancellation between these two terms is to be expected in moist convective systems (e.g., Holton 2004, section. 11.3). The residual between these two terms produces a
0.5 -K warming at low levels $(2<z<6 \mathrm{~km})$ near the center of the merged vortex (Fig. 15e). The vertical eddy heat flux term $(\langle\mathrm{EVPT}\rangle)$ produces a heating maximum in the upper troposphere. This is nearly balanced by the difference in diabatic (latent) heating and adiabatic cooling there ( $\langle$ THDOT $\rangle+\langle$ MVPT $\rangle$; not shown). Although there are small contributions from the mean radial advection $(\langle\mathrm{MRPT}\rangle)$ and radial eddy heat flux $(\langle$ ERPT $\rangle)$, the midlevel warming exhibited in Fig. 15e owes its origin primarily to the latent heat release within the VHTs.

Other diabatic vortex merger events have been examined and the results are qualitatively similar. The maximum heating rate for the VHTs is generally located at midlevels (approximately $z=5-8 \mathrm{~km}$; Fig. 15f). The VHTs consequently generate an abundance of near-surface PV (Raymond and Jiang 1990; ME98). At early times the behavior of vorticity/PV is characterized by frequent small-scale diabatic merger events, each of which appears to stimulate an increase of near-surface 

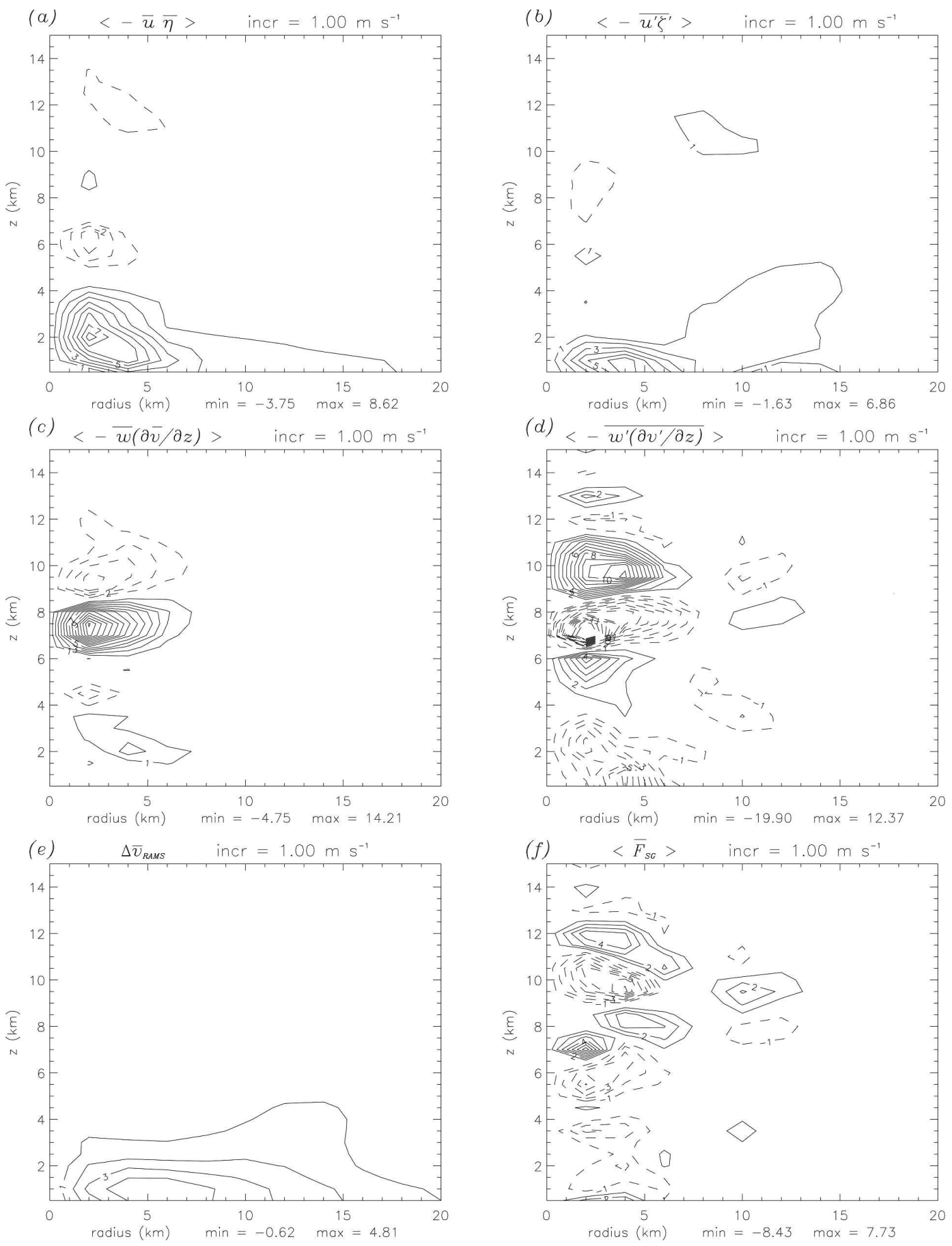

FIG. 14. Time-integrated tendency terms (denoted by $<>$ ) over 40 min from the local mean tangential momentum equation [Eq. (6)] for the diabatic merger event highlighted in Figs. 3 and 12 of control experiment, expt A1. The time integral of (a) $\langle\mathrm{MRAV}\rangle$, (b) $\langle\mathrm{ERRV}\rangle$, (c) $\langle\mathrm{MVTM}\rangle$, and (d) $\langle\mathrm{EVTM}\rangle$. (e) Change in local mean tangential tangential velocity over $40 \mathrm{~min}$ as inferred from the RAMS simulation, $\Delta \bar{v}_{\text {RAMS }}$. (f) The corresponding time integral of the residual subgrid-scale tendency term $\left\langle\bar{F}_{\mathrm{sg}}\right\rangle$ (including surface layer processes). The zero contour is omitted. Negative contours are dashed. 

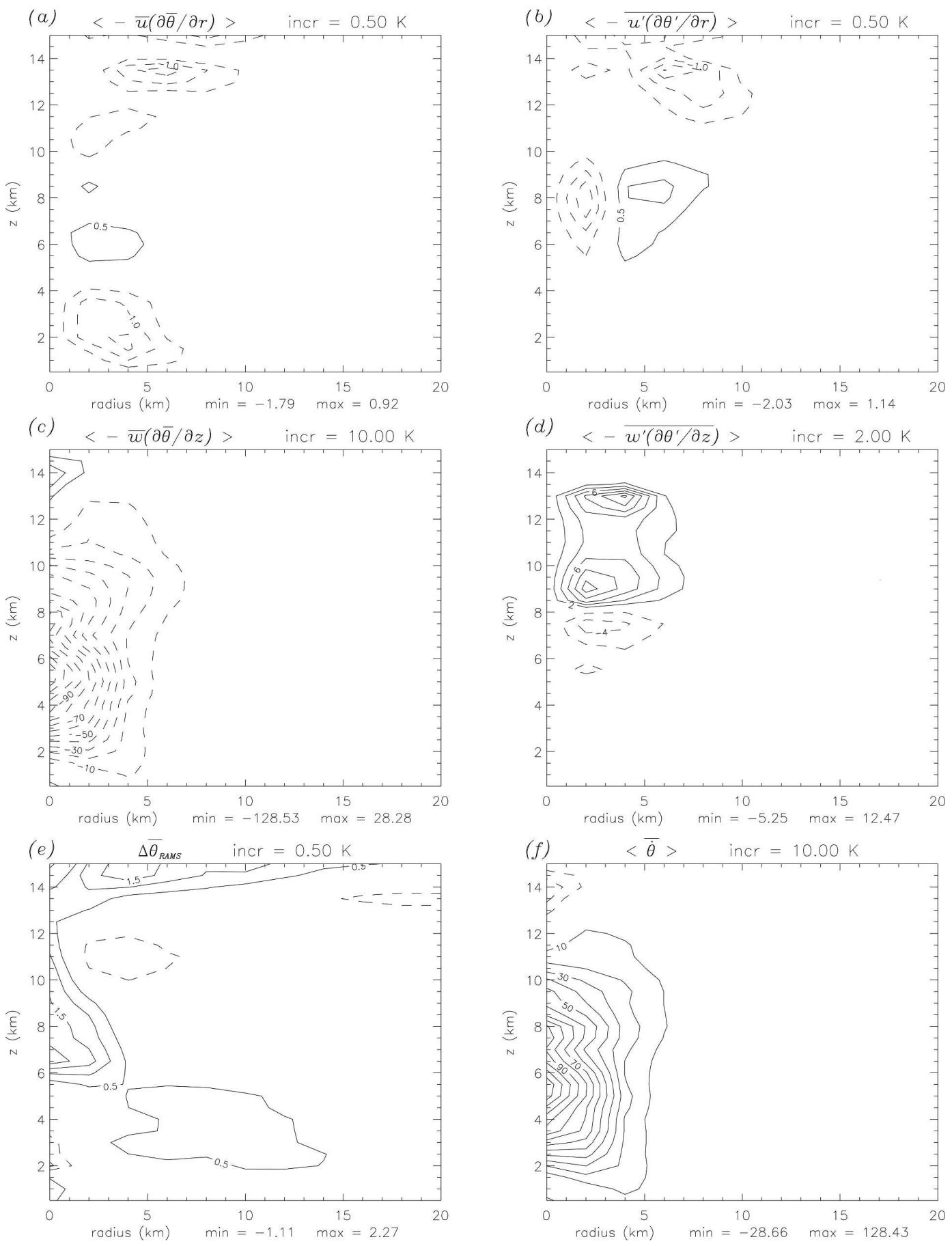

FIG. 15. Time-integrated tendency terms (denoted by $<>$ ) over $40 \mathrm{~min}$ from the local mean potential temperature equation [Eq. (7)] for the diabatic merger event highlighted in Figs. 3 and 12 of the control experiment, expt A1. The time integral of (a) $\langle\mathrm{MRPT}\rangle$, (b) $\langle\mathrm{ERPT}\rangle$, (c) $\langle\mathrm{MVPT}\rangle$, and (d) $\langle\mathrm{EVPT}\rangle$. (e) The 40-min change in the local mean potential temperature as inferred from the RAMS simulation, $\Delta \bar{\theta}_{\text {RAMS }}$. (f) The time integral of $\langle$ THDOT $\rangle$. The zero contour is omitted. Negative contours are dashed.

$\mathrm{PV} /$ vorticity. As time passes, the cumulative nearsurface $\mathrm{PV} /$ vorticity increases even though the individual VHTs lose their convective signature and decay in intensity somewhat. The VHTs and the corre- sponding diabatic vortex mergers serve to vortically prime the mesoscale environment, making the spinup into a tropical depression vortex more likely (Ooyama 1982; H04). 


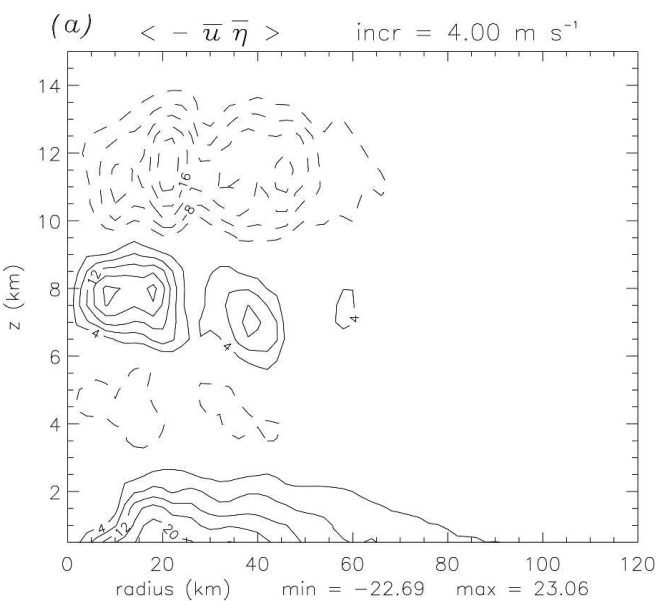

(c) $\left\langle-\bar{w}(\partial \bar{v} / \partial z)>\quad\right.$ incr $=4.00 \mathrm{~m} \mathrm{~s}^{-1}$
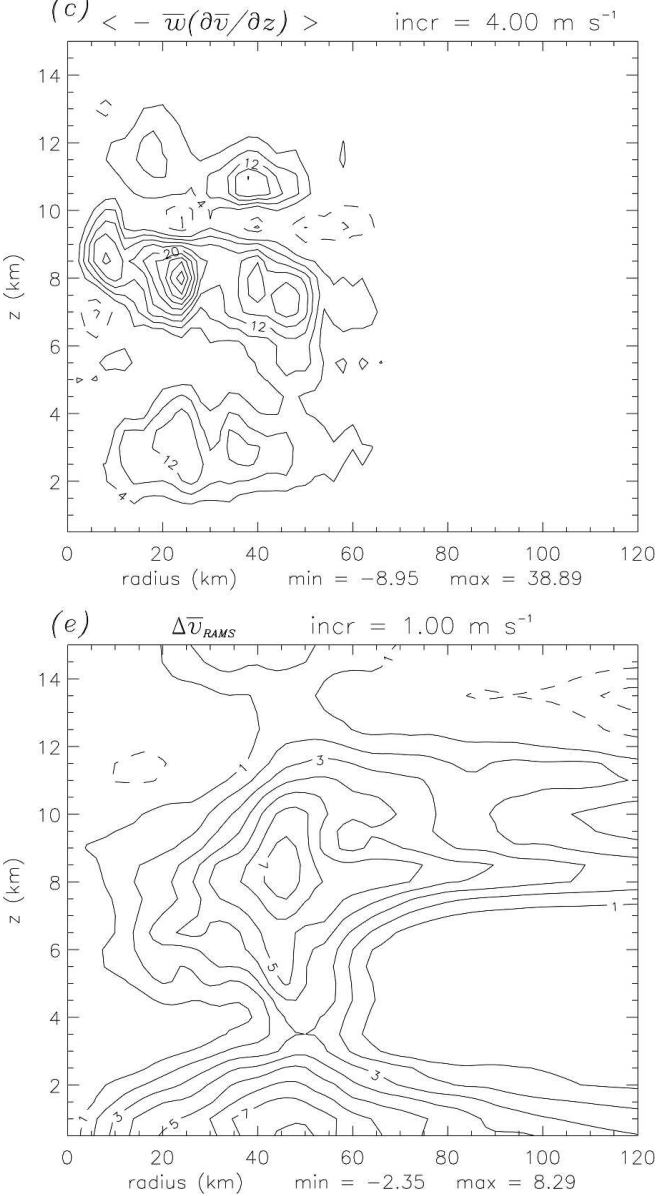

\section{b. System-scale budgets of tangential momentum and potential temperature}

The system-scale tangential momentum budget shown in Fig. 16 suggests a somewhat different picture than its VHT counterpart. The mean terms $(\langle\mathrm{MRAV}\rangle$ and $\langle$ MVTM $\rangle)$ spin up the near-surface $(0<z<2 \mathrm{~km})$ and upper-tropospheric $(6<z<9 \mathrm{~km})$ tangential flow.
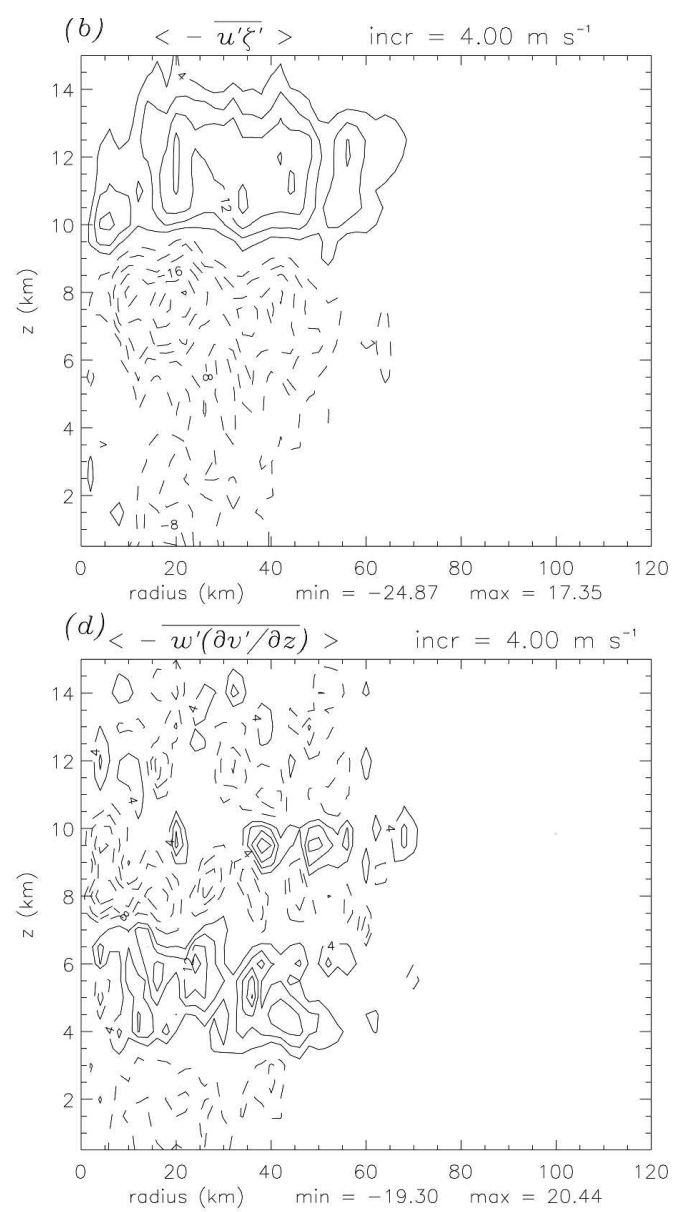

FIG. 16. Time-integrated tendency terms (denoted by $<>$ ) over $24 \mathrm{~h}$ from system-scale tangential momentum equation [Eq. (6)] of control simulation, expt A1. The time integral of (a) $\langle$ MRAV $\rangle$, (b) $\langle$ ERRV $\rangle$, (c) $\langle$ MVTM $\rangle$, and (d) $\langle$ EVTM $\rangle$. (e) The change in the system-scale mean tangential velocity over $24 \mathrm{~h}$ as inferred from the RAMS simulation, $\Delta \bar{v}_{\text {RAMS }}$. The zero contour is omitted. Negative contours are dashed. Note the larger horizontal scale compared to Fig. 14.

Both eddy terms ( $\langle$ ERRV $\rangle$ and $\langle$ EVTM $\rangle$ ), however, tend to spin down the near-surface tangential flow. The opposing tendency between the mean and eddy terms is not an artifact of choosing an improper center of coordinates (the results are found to be insensitive to different coordinate centers). Unlike previous thought experiments in which the convectively generated PV anomalies were added impulsively and then allowed to 

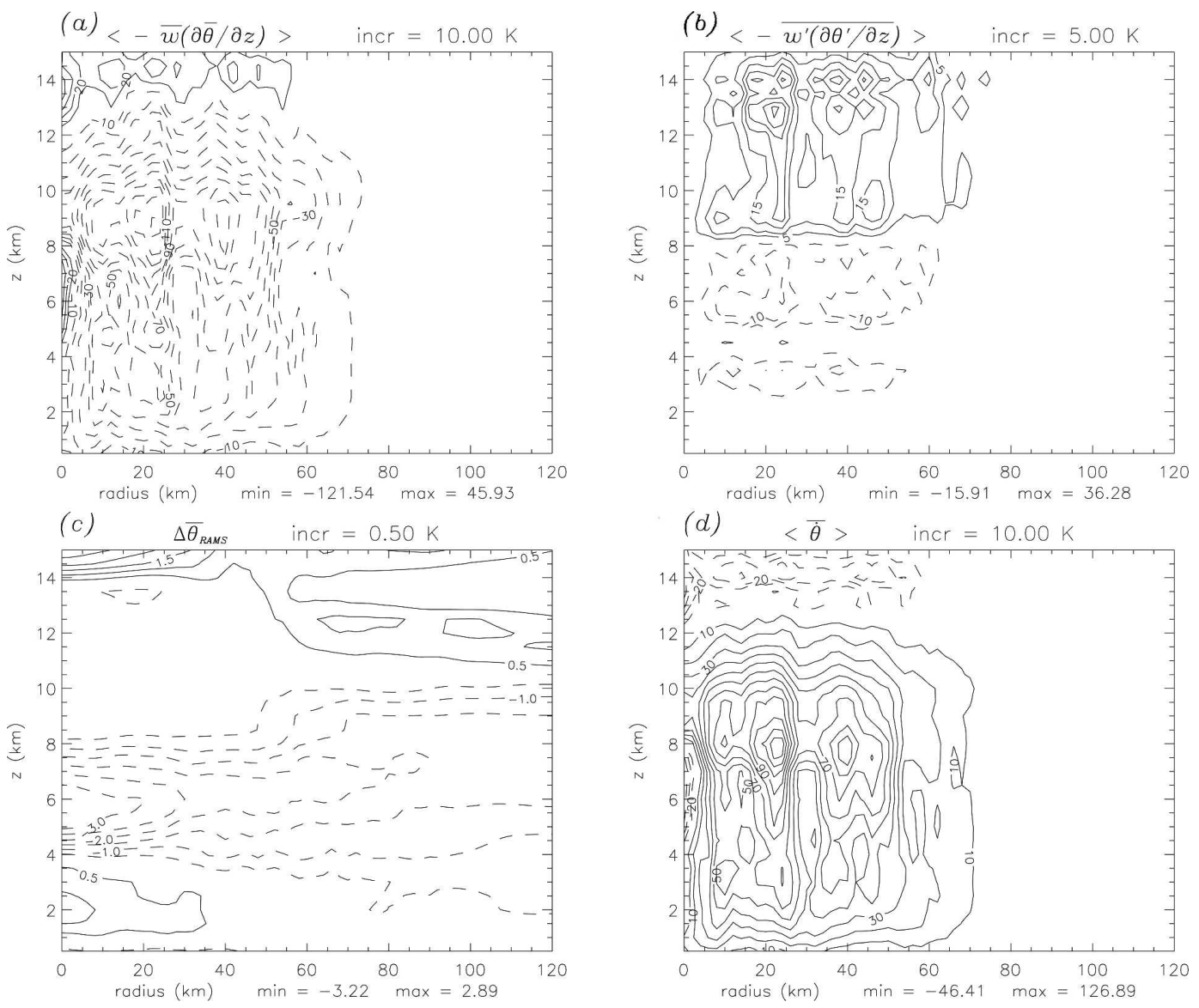

FIG. 17. Time-integrated tendency terms (denoted by $<>$ ) over $24 \mathrm{~h}$ from system-scale potential temperature equation [Eq. (7)] of the control simulation, expt A1. The time integral of (a) $\langle$ MVPT $\rangle$ and (b) $\langle$ EVPT $\rangle$. (c) The 24-h change in the local mean potential temperature inferred from the RAMS simulation, $\Delta \bar{\theta}_{\text {RAMS. }}$ (d) The time integral of $\langle$ THDOT $\rangle$. The zero contour is omitted. Negative contours are dashed. Note the larger horizontal scale compared to Fig. 15.

axisymmetrize under horizontal straining by the parent (mesoscale) vortex (ME98, section 4d; MM00, section 4a; EM01), here the PV anomalies are convectively sustained for characteristic times $\tau \geq O(1 \mathrm{~h})$ and the corresponding near-surface $\mathrm{PV}$ anomalies possess large amplitudes compared to the PV of the parent MCV. When viewed as eddies the intense PV anomalies are then largely able to resist straining by the system-scale flow, and behave effectively as eddy-momentum sinks to the system-scale flow. ${ }^{6}$

The system-scale potential temperature budget shown in Fig. 17 is broadly similar to its VHT counterpart, with the lower tropospheric warming coming pri-

\footnotetext{
${ }^{6}$ In general, however, the VHTs and residual vortices are highly localized structures, see, e.g., Fig. 3. Under such conditions the mean terms $\langle$ MRAV $\rangle$ and $\langle$ MVTM $\rangle$ generally include contributions from the VHTs. See section 7a for more detail.
}

marily from the imbalance between the diabatic heating term $(\langle$ THDOT $\rangle)$ and the mean adiabatic cooling term $(\langle\mathrm{MVPT}\rangle)$. Although the simulations evidence some subsidence warming very near the system center, this warming has a very small magnitude compared to the system-scale latent heating generated by the VHTs.

\section{VHT contribution to system-scale spinup}

The foregoing results demonstrated that on the system scale the near-surface $(0<z<2 \mathrm{~km})$ spinup of tangential winds was due primarily to the mean radial influx of mean absolute vorticity, $-\overline{u \eta}$. What, then, is the relationship during the transformation process between the VHTs and the system-scale influx of mean vorticity?

\section{a. Direct contribution}

The direct contribution of the VHTs to the systemscale mean influx can be clarified by estimating how the 
VHTs contribute to the system-scale absolute vertical vorticity, $\bar{\eta}$. At each vertical level one may decompose the instantaneous absolute vertical vorticity into a sum of the initial vorticity from the parent MCV and vorticity generated by subsequent towers, downdrafts, etc. If, in the time integration of the system-scale radial vorticity flux, one neglects all vertical vorticity anomalies generated by hot tower activity, one obtains a substantially weaker (and hence slower) near-surface spinup for the control run than observed (by approximately $40 \%$, not shown). The reason why the VHTs accelerate the near-surface system-scale spinup is because there is strong cyclonic vortex-tube stretching near the lower surface in association with the VHT updrafts (e.g., Fig. 11). From Eq. (5), the tendency to intensify cyclonic vortices over anticyclonic vortices within the cyclonic MCV embryo (a cyclone/anticyclone asymmetry) yields a turbulent vorticity distribution skewed toward cyclonic vortices.

\section{b. Indirect contribution}

The indirect contribution of the VHTs to the mean influx may be assessed by determining how the VHTs influence the mean radial flow, $\bar{u}$. A simple method employs Eliassen's balanced vortex model (Eliassen 1951) to determine the slowly evolving transverse motions forced by the VHTs (represented here as a superposition of axisymmetric heat and momentum forcing and azimuthally averaged eddy fluxes). For simplicity, we employ a Boussinesq version of Eliassen's balanced vortex model on an $f$ plane in the pseudoheight coordinate $z=\left[1-\left(p / p_{0}\right)^{\kappa}\right]\left(c_{p} \theta_{0} / g\right)$, as defined in Hoskins and Bretherton (1972). ${ }^{7}$

Assuming the axisymmetric motions evolve in hydrostatic and gradient wind balance, the corresponding balance equations are

$$
\begin{aligned}
\frac{\partial \bar{v}}{\partial t}+\overline{u \eta}+\bar{w} \frac{\partial \bar{v}}{\partial z} & =\bar{F}, \\
\frac{\partial^{2} \bar{\phi}}{\partial t \partial z}+\bar{u} \frac{\partial^{2} \bar{\phi}}{\partial r \partial z}+\bar{w} \frac{\partial^{2} \bar{\phi}}{\partial z^{2}} & =\bar{Q}, \\
f \bar{v}+\frac{\bar{v}^{2}}{r} & =\frac{\partial \bar{\phi}}{\partial r}, \\
\frac{\partial \bar{\phi}}{\partial z} & =g \frac{\bar{\theta}}{\theta_{0}} .
\end{aligned}
$$

\footnotetext{
${ }^{7}$ Though the difference between pseudoheight coordinates and geometric coordinates is insignificant during the genesis phase, we will proceed with the pseudoheight nomenclature.
}

Equations (8) and (9) are the mean tangential momentum and mean potential temperature equations, respectively; Eqs. (10) and (11) are the (axisymmetric) gradient wind and hydrostatic balance equations, respectively; and Eq. (11) has been used to express Eq. (9) in terms of the mean flow geopotential. (See Table 2 for variable definitions.) In Eqs. (8)-(11), the heat forcing is defined as $\bar{Q}=g / \theta_{0}\left(-\overline{u^{\prime} \partial \theta^{\prime} / \partial r}-\overline{w^{\prime} \partial \theta^{\prime} / \partial z}+\bar{\theta}\right)$, where the first two terms represent eddy radial and vertical heat fluxes, and $\bar{\theta}$ is the mean diabatic heating rate. The momentum forcing is defined similarly as $\bar{F}=-\overline{u^{\prime} \zeta^{\prime}}$ $\overline{w^{\prime} \partial v^{\prime} / \partial z}+\bar{F}_{\mathrm{sg}}$, where the first two terms represent the eddy radial vorticity and eddy vertical momentum fluxes, and $\bar{F}_{\text {sg }}$ is the term owing to subgrid-scale processes in the numerical model (comprising both diffusive and surface layer processes). As described in appendix B, both $\overline{\dot{\theta}}$ and $\bar{F}_{\text {sg }}$ are calculated as residual terms in the azimuthal mean thermodynamic and tangential momentum equations, respectively.

Insisting that Eqs. (8)-(11) maintain (axisymmetric) thermal wind balance, one obtains the Sawyer-Eliassen equation for the toroidal streamfunction

$$
\begin{gathered}
\frac{\partial}{\partial r}\left(\frac{N^{2}}{r} \frac{\partial \psi}{\partial r}-\frac{\bar{\xi}}{r} \frac{\partial \bar{v}}{\partial z} \frac{\partial \psi}{\partial z}\right)+\frac{\partial}{\partial z}\left(-\frac{\bar{\xi}}{r} \frac{\partial \bar{v}}{\partial z} \frac{\partial \psi}{\partial r}+\frac{\bar{\xi} \bar{\eta}}{r} \frac{\partial \psi}{\partial z}\right) \\
=\frac{\partial \bar{Q}}{\partial r}-\frac{\partial}{\partial z}(\overline{\xi F})
\end{gathered}
$$

The toroidal streamfunction $\psi$ is related to the toroidal circulation $(\bar{u}, \bar{w})$ in the usual way for a Boussinesq

TABLE 2. Definition of variables and constants used in section 7.

\begin{tabular}{ll}
\hline \hline$R$ & Radius \\
$\lambda$ & Azimuthal coordinate \\
$z$ & Vertical (pseudoheight) coordinate \\
$u$ & Radial velocity \\
$v$ & Tangential velocity \\
$w$ & Vertical velocity \\
$f$ & Coriolis parameter (planetary vorticity) \\
$\zeta$ & Relative vertical vorticity \\
$\eta=f+\zeta$ & Absolute vertical vorticity \\
$p$ & Pressure \\
$p_{0}$ & Reference pressure (1000 mb) \\
$\phi$ & Geopotential \\
$\theta$ & Potential temperature \\
$\theta_{0}$ & Reference potential temperature $(300 \mathrm{~K})$ \\
$c_{p}$ & Specific heat of air at constant pressure \\
$R_{d}$ & $\left(1004 \mathrm{~J} \mathrm{~kg}^{-1} \mathrm{~K}^{-1}\right)$ \\
$\kappa$ & Gas constant for dry air (287 $\left.\mathrm{J} \mathrm{kg}^{-1} \mathrm{~K}^{-1}\right)$ \\
$\bar{\eta} \bar{\xi}=(f+\bar{\zeta})$ & $R_{d} / c_{p}$ \\
$N^{2}=\partial^{2} \bar{\phi} / \partial z^{2}$ & Azimuthally averaged inertial (centrifugal) \\
\hline
\end{tabular}



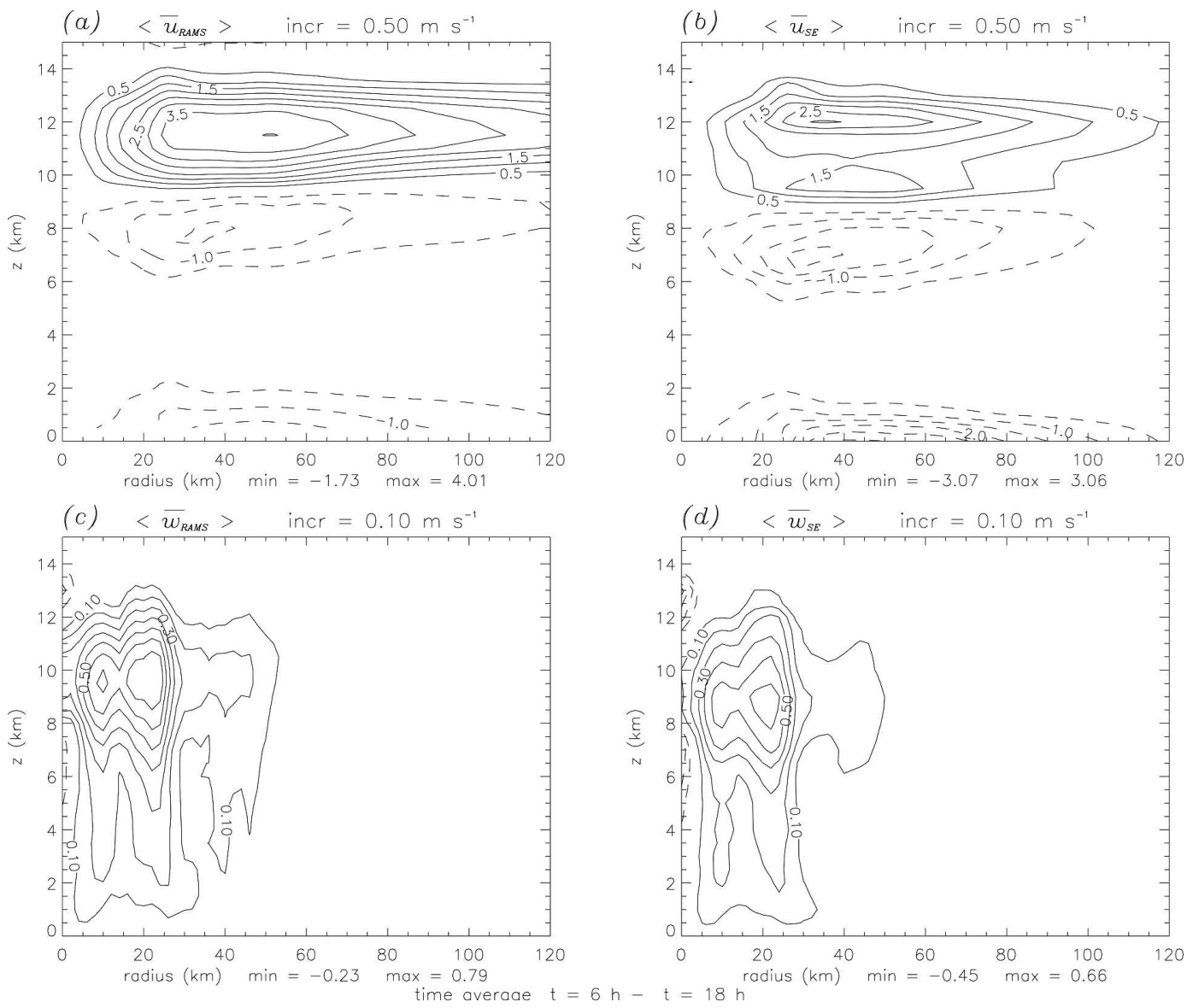

FIG. 18. The 12 -h time average (denoted by $<>$ ) of system-scale (a) RAMS mean radial velocity $\left\langle\bar{u}_{\text {RAMS }}\right\rangle$, (b) Sawyer-Eliassen radial velocity $\left\langle\bar{u}_{\text {SE }}\right\rangle$, (c) RAMS mean vertical velocity $\left\langle\bar{w}_{\text {RAMS }}\right\rangle$, and (d) Sawyer-Eliassen vertical velocity $\left\langle\bar{w}_{\mathrm{SE}}\right\rangle$. Time averages are calculated from $t=6 \mathrm{~h}$ to $t=18 \mathrm{~h}$ from the control simulation, expt A1. The zero contour is omitted. Negative contours are dashed.

fluid: $\bar{u}=-\partial \psi / r \partial z$ and $\bar{w}=\partial \psi / r \partial r$. For the control and sensitivity experiments, the azimuthal mean diabatic heating rate $\dot{\theta}$ dominates other forcing terms in Eq. (12) by one order of magnitude. The boundary condition of zero normal flow along the $r-z$ boundary of the vortex implies that $\psi=0$ along the $r-z$ boundary.

The PDE (12) is elliptic and a unique solution for $\psi$ is guaranteed if the discriminant (solvability) condition

$$
N^{2} \bar{\xi} \bar{\eta}-\left(\bar{\xi} \frac{\partial \bar{v}}{\partial z}\right)^{2}>0
$$

is satisfied throughout the fluid (Shapiro and Willoughby 1982). We solve Eq. (12) using standard successive overrelaxation (SOR; Press et al. 1992). The solvability criterion (13) is violated only locally, and no regularization procedure is needed to obtain a convergent solution during the metamorphosis phase.

\section{c. RAMS versus quasi-balance model}

Figure 18 shows a 12-h time average of the azimuthal mean toroidal (secondary) circulation on the system scale (mesoscale), as diagnosed from the control simulation (Figs. 18a,c), and predicted using the Eliassen balanced vortex model (Figs. 18b,d). ${ }^{8}$ Note the larger horizontal scale compared to Figs. 14 and 15. The averaging period is from $t=6 \mathrm{~h}$ to $t=18 \mathrm{~h}$ in the simulation, a time interval spanning the transformation from the midlevel MCV to a surface-concentrated tropical depression in the control experiment. The mean vertical velocity field predicted by the Eliassen model is qualitatively and quantitatively similar to the corre-

\footnotetext{
${ }^{8}$ Instantaneous comparisons have also been made during the genesis period and the RAMS and Eliassen results show good agreement throughout.
} 
sponding RAMS vertical velocity. The upper-tropospheric $(6<z<14 \mathrm{~km})$ inflow and outflow are also well captured by the Eliassen model. Although the maximum near-surface inflow predicted by the Eliassen model is roughly $70 \%$ stronger than the maximum near-surface inflow observed in the averaged RAMS data, the balanced inflow occurs over a shallower layer and the near-surface $(0<z<2 \mathrm{~km})$ volume flow rate into the incipient TC is approximately the same. Thus, on the system scale, the balance model's toroidal circulation agrees well with that of the RAMS model.

The foregoing results suggest two ways that the VHTs contribute to the upscale organization during the pre-WISHE phase. In an initial cyclonic vorticity environment, the strong latent heat release in the VHTs and the associated vortex tube stretching at low-levels yields a turbulent vorticity field with a cyclonic vorticity bias. The aggregate of VHT cores generate a thermally direct quasi-balanced toroidal circulation on the system scale. The toroidal circulation has two inflow branches: an upper inflow $(6<z<9 \mathrm{~km})$, and a near-surface inflow $(0<z<2 \mathrm{~km})$. Both inflow branches converge cyclonic vorticity of the initial MCV and VHTgenerated cyclonic vorticity anomalies.

\section{Summary and conclusions}

Useful insight into the thermomechanics of tropical cyclogenesis has been obtained by considering the problem of the transformation of an isolated cyclonic $\mathrm{MCV}$ into a surface-concentrated (warm core) tropical depression vortex. Beginning with a midlevel cyclonic MCV with weak cyclonic circulation at the ocean surface and realistic thermodynamic soundings and SSTs, the cloud model simulations point to an upscale organization mechanism operating on realistic time scales that precedes WISHE amplification.

Within the convectively unstable and vorticity-rich environment of the initial MCV embryo, horizontally small-scale cyclonic VHTs emerge as the dominant coherent structures. Although at first sight this may appear similar to now standard large-scale (quasi twodimensional) turbulence phenomenology, the upscale organization mechanism examined here is distinct from "free-decay" turbulence that is constrained by material conservation of PV (e.g., Fornberg 1977; Basdevant et al. 1981; McWilliams 1984; Carnevale et al. 1992; McWilliams et al. 1999). Upscale growth here is punctuated by strongly three-dimensional convective events (VHTs and ensuing convective downdrafts), phase transition processes in which the nonadvective PV flux is comparable to the advective PV flux within the cumulus zone (Haynes and McIntyre 1987), and a corresponding bias in the generation of cyclonic PV anomalies in the lower troposphere.

Given the fact that the simulated VHTs are threedimensional structures possessing height-to-width aspect ratios of order unity and maximum updraft velocities that exceed maximum horizontal swirl velocities, what, then, is the nature of the upscale growth and surface spinup on the system scale? Prior to the WISHE amplification phase, the initial MCV provides a convectively unstable and cyclonic vorticity-rich environment for the intense cyclonic vertical vorticity $\left(\eta>3 \times 10^{-3} \mathrm{~s}^{-1}\right)$ cores that arise via tilting and stretching of preexisting vorticity by the hot tower updrafts. The MCV and vortical cores create a locally protected environment (McWilliams 1984; Julien et al. 1999; Reasor et al. 2004) supporting a more efficient conversion of latent heat energy into rotational (kinetic) energy of the horizontal winds (Schubert and Hack 1982; Hack and Schubert 1986). While individually possessing relatively short lifetimes (on the order of $1 \mathrm{~h}$ ), the aggregate of VHTs (an active population consisting of growing, quasi-steady, merging, and dying towers) mimic a quasi-steady heating rate on the system-scale circulation. For the system scale to remain in thermal wind balance, a thermodynamically direct toroidal circulation is generated with inflow near the surface $(0<z<2 \mathrm{~km})$ and mid- to upper levels $(6<z<9 \mathrm{~km})$, and outflow in the upper troposphere $(9<z<14 \mathrm{~km})$. This quasi-balanced toroidal circulation converges near-surface and mid- to upper-level cyclonic vorticity of the initial MCV and convectivescale cyclonic vorticity anomalies generated by VHT events.

The VHT paradigm presented here for tropical cyclogenesis seems physically plausible. Further tests are encouraged through diagnoses of high-resolution operational and cloud models, examination of disruption scenarios for incipient vortices (dry air intrusions, vertical shear, etc.), and a meteorological field experiment.

Acknowledgments. This work was supported in part by the National Aeronautics and Space Administration (NASA) Fourth Convective and Moisture Experiment (CAMEX) Grant NAG5-11140, the National Science Foundation, under Grant NSF-ATM-0101781, and the Office of Naval Research Grant N00014-02-0474. We are grateful to L. Bosart, C. Davis, R. Elsberry, R. Johnson, F. Marks Jr., J. McWilliams, W. Schubert, K. Tory, and E. Zipser for providing constructive comments during the course of this work. 


\section{APPENDIX A}

\section{List of Model Parameters}

Number of grids

Number of horizontal grid points for grids $1 / 2 / 3$

Number of vertical levels

Horizontal coordinate

Horizontal grid increment for grids $1 / 2 / 3$

Vertical grid increment

Vertical grid stretch ratio

Grid top

Grid time step for grids 1/2/3

Center latitude

Center longitude

Lateral boundary

Lower boundary

Upper boundary

Radiation

Turbulence closure

Cloud microphysics
3

$60 / 90 / 137$

26

Cartesian

$24 \mathrm{~km} / 6 \mathrm{~km} / 2 \mathrm{~km}$

$400 \mathrm{~m}$ at the surface

1.065

$22.6 \mathrm{~km}$

$30 \mathrm{~s} / 10 \mathrm{~s} / 5 \mathrm{~s}$

$15^{\circ}$

$-40^{\circ}$

Klemp and Wilhelmson (1978) radiation condition.

Sea surface temperature for control experiment, 302.15

K. Charnock's constant set to 0.018 in the surface roughness formulation.

Rigid lid with a 5-km-deep high-viscosity layer aloft.

Harrington (1997) long/shortwave model with a tendency update frequency of $900 \mathrm{~s}$.

Smagorinsky (1963) deformation- $K$ closure scheme with stability modifications made by Lilly (1962) and Hill (1974). Value of Smagorinsky's constant set to 0.25 for both horizontal and vertical mixing. Ratio of eddy mixing coefficients for heat and momentum equal to 3.

Single-moment scheme (Walko et al. 1995) with categories for rain, snow, aggregates, graupel, and hail using mean mass diameters of $1.1,0.3,0.5$, and $1 \mathrm{~mm}$, respectively. The value of the shape parameter for the generalized gamma distribution is set to 1 for rain (i.e., Marshall-Palmer) and to 2 for all other categories. A cloud water category is used with a fixed cloud condensation nuclei concentration of $0.2 \times 10^{9}$ $\mathrm{m}^{-3}$. There is also a pristine ice category that has a prognostic equation for number concentration (twomoment scheme; Meyers et al. 1997).

$500 \mathrm{~m}$. The interpolation occurs prior to other calculations performed during postprocessing.

\section{Diagnostic Package}

The local-scale and system-scale dynamics are analyzed from a traditional vortex-centric perspective, transforming the Cartesian model data into a local cylindrical coordinate system centered on particular events of physical interest. Because of the grid stagger in the RAMS model output, the Cartesian $x$ - and $y$-grid velocities are first interpolated to the thermodynamic variable grid points at each vertical level. All data are then interpolated to vertical grid levels evenly spaced at

\section{a. Determining system center and azimuthal averaging}

For the first $24 \mathrm{~h}$ of each simulation the center of the domain is utilized as the system-scale center. Once the surface pressure anomaly becomes well defined (persistent), the system-scale center is calculated by using a "best circle fit" method that first approximates a given surface pressure contour as a circle and uses the center of the circle as the system center. The resulting center 
is kept constant with height. This approach is believed superior to using the location of minimum surface pressure because, at early times, the minimum surface pressure is typically found within VHT cores whose lifetimes are short (order $1 \mathrm{~h}$ ) compared to the evolutionary time scale of the system-scale vortex.

The cylindrical grid used consists of 25 azimuthal points. The number of radial points is dependent on the location of the storm center. The radial grid spacing is uniform with a grid increment equal to the Cartesian horizontal grid spacing of the finest grid. Once a center is established, scalar fields are interpolated to the cylindrical grid. Vector fields, such as the radial and tangential wind components, are calculated by applying a rotation transformation to the Cartesian vector at each grid point. The tangential and radial velocities are then interpolated onto the cylindrical grid. Azimuthal averages are calculated as an arithmetic average of values along a constant radius, and are denoted with an overbar. Deviations from the azimuthal average are denoted with primes.

\section{b. Tangential momentum and potential temperature budgets}

The vortex-centric model is used to obtain local and system-scale budgets of azimuthal mean tangential momentum [Eq. (6)] and azimuthal mean potential temperature [Eq. (7)]. Rather than compute the diabatic heating rate $\dot{\theta}$ by adding the microphysical, radiation, and subgrid-scale tendencies to the right-hand side of Eq. (7), we calculate the heating rate via residual, $\dot{\theta}=$ $\partial \theta / \partial t+\mathbf{u} \cdot \boldsymbol{\nabla} \theta$. The residual method has the same order of accuracy as the former, and is more convenient to compute since the RAMS model predicts ice-liquid potential temperature (Tripoli and Cotton 1981) and then diagnoses the potential temperature and water vapor mixing ratio. A similar residual method is used to calculate $\bar{F}_{s g}$ in (6).

\section{REFERENCES}

Anthes, R. A., 1982: Tropical Cyclones: Their Evolution, Structure, and Effects. Meteor. Monogr., No. 19, Amer. Meteor. Sco., 208 pp.

- 2003: Hot towers and hurricanes: Early observations theories, and models. Cloud Systems, Hurricanes, and the Tropical Rainfall Measuring Mission (TRMM), Meteor. Monogr., No. 51, Amer. Meteor. Soc., 139-148

Basdevant, C., B. Legras, R. Sadourny, and M. Béland, 1981: A study of barotropic model flows: Intermittency, waves, and predictability. J. Atmos. Sci., 38, 2305-2326.

Bister, M., and K. Emanuel, 1997: The genesis of Hurricane Guillermo: TEXMEX analyses and a modeling study. Mon. Wea. Rev., 125, 2662-2682.
Bolton, D., 1980: The computation of equivalent potential temperature. Mon. Wea. Rev., 108, 1046-1053.

Braun, S. A., 2002: A cloud-resolving simulation of Hurricane Bob (1991): Storm structure and eyewall buoyancy. Mon. Wea. Rev., 130, 1573-1592.

— - M. T. Montgomery, and Z. Pu, 2006: High-resolution simulation of Hurricane Bonnie (1998). Part I: The organization of eyewall vertical motion. J. Atmos. Sci., 63, 19-42

Bryan, G. H., J. C. Wyngaard, and J. M. Fritsch, 2003: Resolution requirements for the simulation of deep moist convection. Mon. Wea. Rev., 131, 2394-2416.

Carnevale, G. F., J. C. McWilliams, Y. Pomeau, J. B. Weiss, and W. R. Young, 1992: Rates, pathways, and end states of nonlinear evolution in decaying two-dimensional turbulence: Scaling theory versus selective decay. Phys. Fluids A, 4, 13141316.

Charney, J. G., and A. Eliassen, 1964: On the growth of the hurricane depression. J. Atmos. Sci., 21, 68-75.

Charnock, H., 1955: Wind stress on a water surface. Quart. J. Roy. Meteor. Soc., 81, 639-640.

Clark, T. L., and R. D. Farley, 1984: Severe downslope windstorm calculations in two and three spatial dimensions using anelastic grid nesting: A possible mechanism for gustiness. $J$. Atmos. Sci., 41, 329-350.

Cotton, W. R., and R. A. Anthes, 1989: Storm and Cloud Dynamics. Academic Press, 883 pp.

- , and Coauthors, 2003: RAMS 2001: Current status and future directions. Meteor. Atmos. Phys., 82, 5-29.

Cram, T. A., M. T. Montgomery, and R. F. A. Hertenstein, 2002: Early evolution of vertical vorticity in a numerically simulated idealized convective line. J. Atmos. Sci., 59, 2113-2127.

Davis, C. A., and L. F. Bosart, 2001: Numerical simulations of the genesis of Hurricane Diana (1984). Part I: Control simulation. Mon. Wea. Rev., 129, 1859-1881.

Dunion, J. P., and C. S. Velden, 2004: The impact of the Saharan air layer on Atlantic tropical cyclone activity. Bull. Amer. Meteor. Soc., 85, 353-365.

Eliassen, A., 1951: Slow thermally or frictionally controlled meridional circulation in a circular vortex. Astrophys. Norv., 5, $19-60$.

_ , and M. Lystad, 1977: The Ekman layer of a circular vortex: A numerical and theoretical study. Geophys. Norv., 31, 1-16.

Enagonio, J., and M. T. Montgomery, 2001: Tropical cyclogenesis via convectively forced vortex Rossby waves in a shallow water primitive equation model. J. Atmos. Sci., 58, 685-706.

Fornberg, B., 1977: A numerical study of 2-D turbulence. J. Comput. Phys., 25, 1-31.

Fulton, J. D., 2001: Insights into the dynamics of the hurricane vortex using a high resolution numerical simulation of Hurricane Bob (1991). M.S. thesis, Dept. of Atmospheric Science, Colorado State University, 84 pp.

Gray, W. M., 1968: Global view of the origins of tropical disturbances and storms. Mon. Wea. Rev., 96, 669-700.

_, 1998: The formation of tropical cyclones. Meteor. Atmos. Phys., 67, 37-69.

Guinn, T. A., and W. H. Schubert, 1993: Hurricane spiral bands. $J$. Atmos. Sci., 50, 3380-3403.

Hack, J. J., and W. H. Schubert, 1986: Nonlinear response of atmospheric vortices to heating by organized cumulus convection. J. Atmos. Sci., 43, 1559-1573.

Harr, P. A., and R. L. Elsberry, 1996: Structure of a mesoscale convective system embedded in Typhoon Robyn during TCM-93. Mon. Wea. Rev., 124, 634-652. 
—, M. S. Kalafsky, and R. L. Elsberry, 1996: Environmental conditions prior to formation of a midget tropical storm during TCM-93. Mon. Wea. Rev., 124, 1693-1710.

Harrington, J. Y., 1997: The effects of radiative and microphysical processes on simulated warm and transition season arctic stratus. Ph.D. thesis, Colorado State University, 289 pp.

Haynes, P. H., and M. E. McIntyre, 1987: On the evolution of vorticity and potential vorticity in the presence of diabatic heating and frictional or other forces. J. Atmos. Sci., 44, 828841.

Hendricks, E. A., M. T. Montgomery, and C. A. Davis, 2004: On the role of "vortical" hot towers in formation of tropical cyclone Diana (1984). J. Atmos. Sci., 61, 1209-1232.

Hill, G. E., 1974: Factors controlling the size and spacing of cumulus clouds as revealed by numerical experiments. J. Atmos. Sci., 31, 646-673.

Holton, J. R., 2004: An Introduction to Dynamic Meteorology. 4th ed. Elsevier Academic Press, 529 pp.

Hoskins, B. J., and F. P. Bretherton, 1972: Atmospheric frontogenesis models: Mathematical formulation and solution. J. Atmos. Sci., 29, 11-37.

— - M. E. McIntyre, and A. W. Robertson, 1985: On the use and significance of isentropic potential vorticity maps. Quart. J. Roy. Meteor. Soc., 111, 877-946.

Jordan, C. L., 1958: Mean soundings for the West Indies area. J. Meteor., 15, 91-97.

Jorgensen, D. P., 1984: Mesoscale and convective-scale characteristics of mature hurricanes. Part II: Inner core structure of Hurricane Allen (1980). J. Atmos. Sci., 41, 1287-1311.

Julien, K., S. Legg, J. McWilliams, and J. Werne, 1999: Plumes in rotating convection. Part 1: Ensemble statistics and dynamical balances. J. Fluid Mech., 391, 151-187.

Klemp, J. B., 1987: Dynamics of tornadic thunderstorms. Annu. Rev. Fluid Mech., 19, 369-402.

_ dimensional convective storm dynamics. J. Atmos. Sci., 35, 1070-1086.

Kurihara, Y., and R. E. Tuleya, 1981: A numerical simulation study on the genesis of a tropical storm. Mon. Wea. Rev., 109, 1629-1653.

Lander, M. A., 1994: Description of a monsoon gyre and its effect on the tropical cyclones in the western North Pacific during August 1991. Wea. Forecasting, 9, 640-654.

Legg, S., J. McWilliams, and J. Gao, 1998: Localization of deep ocean convection by a mesoscale eddy. J. Phys. Oceanogr., 28, 944-970.

Lilly, D. K., 1960: On the theory of disturbances in a conditionally unstable atmosphere. Mon. Wea. Rev., 88, 1-17.

_ 1962: On the numerical simulation of buoyant convection. Tellus, 14, 148-172.

Louis, J. F., 1979: A parametric model of vertical eddy fluxes in the atmosphere. Bound.-Layer Meteor., 17, 187-202.

Malkus, J. S., and H. Riehl, 1960: On the dynamics and energy transformations in steady-state hurricanes. Tellus, 12, 1-20.

Mapes, B. E., and R. A. Houze, 1995: Diabatic profiles on western Pacific convective systems. J. Atmos. Sci., 52, 1807-1828.

McIntyre, M. E., 1993: Isentropic distributions of potential vorticity and their relevance to tropical cyclone dynamics. Tropical Cyclone Disasters: Proceedings of ISCU/WMO International Symposium, J. Lighthill et al., Eds., Peking University Press, 143-156.

McWilliams, J. C., 1984: The emergence of isolated coherent vortices in turbulent flow. J. Fluid Mech., 140, 21-43.
— J. B. Weiss, and I. Yavneh, 1999: The vortices of homogeneous geostrophic turbulence. J. Fluid Mech., 401, 1-26.

- L. P. Graves, and M. T. Montgomery, 2003: A formal theory for vortex Rossby waves and vortex evolution. Geophys. Astrophys. Fluid Dyn., 97, 275-309.

Melander, M. V., N. J. Zabusky, and J. C. McWilliams, 1988: Symmetric vortex merger in two dimensions: Causes and conditions. J. Fluid Mech., 195, 303-340.

Meyers, M. P., R. L. Walko, J. Y. Harrington, and W. R. Cotton, 1997: New RAMS cloud microphysics parameterization. Part II: The two-moment scheme. Atmos. Res., 45, 3-39.

Molemaker, M. J., and H. A. Dykstra, 2000: Stability of a cold core eddy in the presence of convection: Hydrostatic versus nonhydrostatic modeling. J. Phys. Oceanogr., 30, 475-494.

Molinari, J., D. Vollaro, and K. L. Corbosiero, 2004: Tropica storm formation in a sheared environment. J. Atmos. Sci., 61, 2493-2509.

Möller, J. D., and M. T. Montgomery, 2000: Tropical cyclone evolution via potential vorticity anomalies in a three-dimensional balance model. J. Atmos. Sci., 57, 3366-3387.

Montgomery, M. T., and R. J. Kallenbach, 1997: A theory for vortex Rossby waves and its application to spiral bands and intensity changes in hurricanes. Quart. J. Roy. Meteor. Soc., 123, 435-465.

, and J. Enagonio, 1998: Tropical cyclogenesis via convectively forced vortex Rossby waves in a three-dimensional quasigeostrophic model. J. Atmos. Sci., 55, 3176-3207.

, H. D. Snell, and Z. Yang, 2001: Axisymmetric spindown dynamics of hurricane-like vortices. J. Atmos. Sci., 58, 421435 .

Nezlin, M. V., and E. N. Snezhkin, Eds., 1993: Rossby Vortices, Spiral Structures, Solitons: Astrophysics and Plasma Physics in Shallow Water Experiments. Springer-Verlag, 223 pp.

Nicholls, M. E., R. A. Pielke Sr., S. Greco, and D. Emmitt, 2000: An investigation of the impact of including CAMEX-3 data on high resolution Hurricane Bonnie simulations. Proc. 24th Conf. on Hurricanes and Tropical Meteorology, Ft. Lauderdale, FL, Amer. Meteor. Soc., 362-363.

Ooyama, K. V., 1964: A dynamical model for the study of tropical cyclone development. Geofis. Int., 4, 187-198.

, 1969: Numerical simulation of the life cycle of tropical cyclones. J. Atmos. Sci., 26, 3-40.

, 1982: Conceptual evolution of the theory and modeling of the tropical cyclone. J. Meteor. Soc. Japan, 60, 369-379.

Palmen, E., and C. W. Newton, 1969: Atmospheric Circulation Systems: Their Structure and Physical Interpretation. Academic Press, 603 pp.

Pielke, R. A., and Coauthors, 1992: A comprehensive meteorological modeling system-RAMS. Meteor. Atmos. Phys., 49, 69-91.

Press, W. H., S. A. Teukolsky, W. T. Vetterling, and B. P. Flannery, 1992: Numerical Recipes in FORTRAN 77: The Art of Scientific Computing. Vol. 1. 2d ed. Cambridge University Press, $933 \mathrm{pp}$.

Raymond, D. J., and H. Jiang, 1990: A theory for long-lived mesoscale convective systems. J. Atmos. Sci., 47, 3067-3077.

_ _ C. López-Carrillo, and L. López Cavazos, 1998: Case-studies of developing east Pacific easterly waves. Quart. J. Roy. Meteor. Soc., 124, 2005-2034.

Reasor, P. D., M. T. Montgomery, and L. D. Grasso, 2004: A new look at the problem of tropical cyclones in vertical shear flow: Vortex resiliency. J. Atmos. Sci., 61, 97-116.

,-- , and L. Bosart, 2005: Mesoscale observations of the 
genesis of Hurricane Dolly (1996). J. Atmos. Sci., 62, 31513171

Reed, R. J., D. C. Norquist, and E. E. Recker, 1977: The structure and properties of African wave disturbances as observed during phase III of GATE. Mon. Wea. Rev., 105, 317-333.

Riehl, H., and J. S. Malkus, 1958: On the heat balance in the equatorial trough zone. Geophysica, 6, 503-538.

Ritchie, E. A., 2003: Some aspects of midlevel vortex interaction in tropical cyclogenesis. Cloud Systems, Hurricanes, and the Tropical Rainfall Measuring Mission (TRMM), Meteor. Monogr., No. 51, Amer. Meteor. Soc., 165-174.

—_, and G. Holland, 1997: Scale interaction during the formation of typhoon Irving. Mon. Wea. Rev., 125, 1377-1396.

—, J. Simpson, W. T. Liu, J. Halverson, C. S. Velden, K. F. Brueske, and H. Pierce, 2002: Present day satellite technology for hurricane research: A closer look at formation and intensification. Hurricane! Coping with Disaster, R. Simpson, Ed., Amer. Geophys. Union, 249-289.

Rotunno, R., and K. Emanuel, 1987: An air-sea interaction theory for tropical cyclones. Part II: Evolutionary study using a nonhydrostatic axisymmetric numerical model. J. Atmos. Sci., 44, $542-561$.

Schubert, W. H., and J. J. Hack, 1982: Inertial stability and tropical cyclone development. J. Atmos. Sci., 39, 1687-1697.

Shapiro, L. J., and H. Willoughby, 1982: The response of balanced hurricanes to local sources of heat and momentum. J. Atmos. Sci., 39, 378-394.

— , and M. T. Montgomery, 1993: A three-dimensional balance theory for rapidly rotating vortices. J. Atmos. Sci., 50, 33223335.

Simpson, J., J. B. Halverson, B. S. Ferrier, W. A. Petersen, R. H. Simpson, R. Blakeslee, and S. L. Durden, 1998: On the role of "hot towers" in tropical cyclone formation. Meteor. Atmos. Phys., 67, 15-35.
Smagorinsky, J. S., 1963: General circulation experiments with the primitive equations. I: The basic experiment. Mon. Wea. Rev., 91, 99-164.

Smith, R. K., 1997: On the theory of CISK. Quart. J. Roy. Meteor. Soc., 123, 407-418.

Trier, S. B., C. A. Davis, and W. C. Skamarock, 2000: Long-lived mesoconvective vortices and their environment. Part II: Induced thermodynamic destabilization in idealized simulations. Mon. Wea. Rev., 128, 3396-3412.

Tripoli, G. J., and W. R. Cotton, 1981: The use of ice-liquid water potential temperature as a thermodynamic variable in deep atmospheric models. Mon. Wea. Rev., 109, 1094-1102.

—_, and — , 1982: The Colorado State University threedimensional cloud/mesoscale model-1982. Part I: General theoretical framework and sensitivity experiments. J. Rech. Atmos., 16, 185-220.

Walko, R. L., W. R. Cotton, J. L. Harrington, and M. P. Myers, 1995: New RAMS cloud microphysics parameterization. Part I: The single-moment scheme. Atmos. Res., 38, 29-62.

Wang, Y., and G. Holland, 1995: On the interaction of tropicalcyclone-scale vortices. IV: Baroclinic vortices. Quart. J. Roy. Meteor. Soc., 121, 95-126.

Zehr, R., 1992: Tropical cyclogenesis in the western North Pacific. NOAA Tech. Rep. NESDIS 61, 181 pp.

Zipser, E. J., 1977: Mesoscale and convective-scale downdrafts as distinct components of squall-line structure. Mon. Wea. Rev., 105, 1568-1589.

—, and C. Gautier, 1978: Mesoscale events within a GATE tropical depression. Mon. Wea. Rev., 106, 789-805.

_ 2003: Some views on "hot towers" after 50 years of tropical field programs and two years of TRMM data. Cloud Systems, Hurricanes, and the Tropical Rainfall Measuring Mission (TRMM), Meteor. Monogr., No. 51, Amer. Meteor. Soc., 4958. 